Predicting Linear and Nonlinear Time Series with Applications in Nuclear Safeguards and Nonproliferation 


\section{Edited by Paul W. Henriksen, Group CIC-1 \\ Prepared by Sharon Hurdle, Group NIS-7}

This work was supported by Defense Programs and the DOE Office of Arms Control and Nonproliferation.

\section{An Affirmative Action/Equal Opportunity Employer}

This report was prepared as an account of work sponsored by an agency of the United States Government. Neither The Regents of the University of California, the United States Government nor any agency therefof, nor any of their employees, makes any warranty, express or implied, or assimes any legal liability or responsibility for the accuracy, completeness, or usefulness of any information, apparatus, product, or process disclosed, or represents that its use would not infringe privately ouned rights. Reference herein to any specific commercial product, process, or seroice by trade name, trademark, mamufacturer, or otherreise, does not necessarily constitute or imply its endorsement, recommendation, or fancoring by The Regents of the University of California, the United States Government, or any agency the refo. The views and opinions of authors expressed herein do not necessarily state or reflect those of The Regents of the University of Californa, the Linited States Government, or any agency thereof. 
Predicting Linear and Nonlinear Time

Series with Applications in Nuclear

Safeguards and Nonproliferation

Tom L. Burr 


\title{
PREDICTING LINEAR AND NONLINEAR TIME SERIES WITH APPLICATIONS IN NUCLEAR SAFEGUARDS AND NONPROLIFERATION
}

\author{
by
}

Tom L. Burr

\begin{abstract}
This report is a primer on the analysis of both linear and nonlinear time series with applications in nuclear safeguards and nonproliferation. We analyze eight simulated and two real time series using both linear and nonlinear modeling techniques. The theoretical treatment is brief but references to pertinent theory are provided. Forecasting is our main goal. However, because our most common approach is to fit models to the data, we also emphasize checking model adequacy by analyzing forecast errors for serial correlation or nonconstant variance.
\end{abstract}

\subsection{Introduction}

This report is intended to be a brief primer on the analysis of both linear and nonlinear time series. A time series is a collection of observations made sequentially over time. A familiar example is the weekly Dow Jones Industrial Average. We only consider time series that are observed at equal intervals of time.

The emphasis in this report will be on using long time series ( 100 or more observations recorded over time) to predict future values of the same series. For that reason, all analyses will be in the time domain rather than the frequency domain. Also, analyses in the time domain are usually considered to be simpler than analyses in the frequency domain. The frequency domain offers advantages for studying the cyclic properties of a time series, which will not concern us here. See Ref. 1 for an introduction to analyses in both time and frequency domains.

The ability to predict future values affords us also the ability to monitor the sequence for anomalous data, which is of interest in nuclear safeguards and nonproliferation. We define anomalous data to be data that is unexplainable by our current knowledge of the system generating the data. If the difference between an observed value and its predicted value is larger than expected considering inadequacies in the model and random error, then that observation is anomalous. Such an observation is also known as an outlier in statistical terminology. In this sense, monitoring data 
for outliers has a long history in statistics, particularly in the area of quality control. However, the technical details of developing schemes to quantify what is meant by "anomalous" continue to bring new challenges. One major issue is whether there is anomalous data in the data we use to build an empirical model. The data we use to build a model is called the training data. The data we use to test a model is called the testing data. This report will only consider the case in which there are no outliers in the training data. The goal is then to make the best use of the training data to monitor the testing data for outliers.

The subject of linear time series is very mature, having its first formal treatment with Yule's autoregressive models in 1927. And certainly informal analysis of both linear and nonlinear time series began well before that. The subject of nonlinear time series is less mature, but during the 1980s and early 1990s there has been considerable activity in nonlinear modelling of time series. Some of the activity has arisen because of the rather curious behavior known as chaos, which is a feature of certain nonlinear time series. This report does not address any of the chaotic behavior of certain nonlinear functions. Rather, the goal is to analyze observed time series explicitly for the purpose of predicting future values. We will introduce several simple but effective techniques for predicting future values that might in cases suggest that the nonlinear function generating the series has chaotic properties, but there will be no emphasis on characterizing the chaotic properties of a time series.

This report is organized as follows. In Sec. 2 we present examples to illustrate techniques to separately analyze the long- and short-term properties of either a linear or a nonlinear time series. In Sec. 3 we describe the linear and nonlinear modelling of the long- and short-term properties of time series. In Sec. 4 we apply linear and nonlinear modelling to several simulated data sets. In

Sec. 5 we apply linear and nonlinear modelling to two real data sets. It is not difficult to construct examples where both the linear and nonlinear modelling strategies described in Sec. 3 will be deficient. Therefore, also in Sec. 5 we introduce three promising nonlinear modelling strategies that generalize the nonlinear modelling strategy introduced in Sec. 3. Section 6 is a summary.

\subsection{Long- and Short-Term Properties of Time Series}

It is probably fair to say that there are two major categories of activity in the analysis of a time series. Refer to Figs. 1 and 2 for the following discussion.

The two categories are trend analysis and local dependency analysis. The time series shown in Fig. 1a exhibits both a trend and a local dependency. The trend appears to be linear or perhaps quadratic. The local dependency is difficult to discern but could explain some of the bumpiness of the graph. The trend is considered a long-term property. The local dependency is a short-term property that refers to the impact that a particular value has on only the next few values of the series.

One of the most useful tools for trend analysis is a simple plot of the series $x_{1}, x_{2}, \ldots, x_{t}$ versus time. Any trend will be apparent in such a plot, as we see in Fig. 1a. 
Onic of the most useful tools in the local-dependency analysis of a linear time series is the sample auto-correlation function (ACF) at lag $k, r(k)$. Let $\bar{x}$ denote the sample mean of the time series and le: $r(k)$ be the estimate, say $\hat{\rho}(k)$, of the true ACF, say $\rho(k)$. A typical definition of $r(k)$ is

$$
r(k)=\frac{\sum_{t=1}^{n-k}\left(x_{t}-\bar{x}\right)\left(x_{t+k}-\bar{x}\right)}{\sum_{t=1}^{n}\left(x_{t}-\bar{x}\right)^{2}} .
$$

Recall that the correlation between any two random variables is a measure of their linear dependence. The sample $\mathrm{ACF}$ at lag $k$ of a time series is a measure of the linear dependence between $x_{t}$ and $x_{t-k}$. If the dependence between $x_{t}$ and $x_{t-k}$ is not linear, then the sample ACF might not be adequate. We introduce another tool to quantify the dependence between $x_{t}$ and $x_{t-k}$ in Sec. 4.

Concerning the sample ACF, note that $r(0)=1$, which conveys the obvious fact that the series is perfectly correlated with itself. For lags $k=1,2, \ldots$, any realistic time series will have $-1<r(k)<1$. Suppose that $r(1)>0$. In view of Equation (1), this means that successive observations tend to be on the same side of the mean. Suppose that $r(1) \approx 1$. Then not only do successive observations tend to be on the same side of the mean, but they also tend to be nearly equal.

In Fig. 1a, because of the strong trend, we expect successive observations to usually be either both above or both below the mean. Consequently, even if there is no local dependence between $x_{t}$ and $x_{t-k}$, the sample ACF will be close to one for the first few lags. Therefore, the analysis of local dependencies is rendered meaningless as long as the long-term trend is present. See Fig. 1b, where the sample ACF remains large for large lags, which suggests that the series contains a trend. Of course, the trend is obvious in the simple time-sequence plot of the series itself in Fig. 1a. In short, it is important to realize that a long-term trend will tend to conceal local effects. Therefore, if local dependencies are to be analyzed, the trend must somehow be removed.

The easiest way to separately analyze long- and short-term dependencies is to appropriately transform the series until its propertiss appear to be constant over time. The properties of primary concern are the mean, variance, and covariance. Formally, we say that a series $x_{1}, x_{2}, \ldots, x_{t}$ is stationary if the probability distribution of $x_{t_{1}}, x_{t_{2}}, \ldots, x_{t_{k}}$ is the same as the probability distribution of $x_{t_{1+\tau}}, x_{t_{2+\tau}}, \ldots, x_{t_{k+\tau}}$ for any $\tau$ and for any subset $x_{t_{1}}, x_{t_{2}}, \ldots, x_{t_{k}}$. Less formally, a series is stationary if the mean $E\left\{x_{t}\right\}$ and the variance $E\left\{\left(x_{t}-E\left\{x_{t}\right\}^{2}\right)\right\}$ of the series do not depend on the time $t$, and if the covariance

$$
\gamma(k)=E\left\{\left(x_{t+k}-E\left\{x_{t}\right\}\right)\left(x_{t}-E\left\{x_{t}\right\}\right)\right\}
$$


depends only on the lag $k$ and not on $t$. (The notation $E\{\cdot\}$ denotes expected value.) The ACF is the scaled auto-covariance:

$$
\rho(k)=\gamma(k) / \rho(0)
$$

A second property required in the formal analysis of a time series is ergodicity. The idea is that we hope to use a single realization of the process to estimate the behavior of a large collection of long sequences from the same probability distribution. The statistician's viewpoint is that a black box is generating the series, and the goal is to describe and estimate what is in the black box. The black box represents the probability distribution that is generating the series. Ergodicity is the condition that must hold if we are to successfully estimate the behavior of a large collection, or ensemble, of series generated from the same black box. Rather than formally define ergodicity, we simply give an example of a series that is not ergodic and an example of a series that is ergodic.

Suppose the black box (probability distribution) that is generating the series operates as follows:

Draw an observation $x_{1}$ according to the standard normal $N(0,1)$ distribution. That is, the first observation of the series $x_{1}$ has a normal distribution with mean 0 and variance 1 . Let $x_{2}=x_{1}$, and in general, $x_{i}=x_{i-1}$ for $i=2,3, \ldots, t$. That is, each of the $t$ observations will equal the first observation so it is a rather uninteresting time series, which would appear to be stationary. However, it is not ergodic because we cannot use this single realization to estimate the behavior of an ensemble of similarly generated series. To see why not, note that the average over time of the $t$ values is simply $x_{1}$, and the sample variance is 0 . This is true for all values of $t$. If it were ergodic, then the time average would converge to the ensemble average as $t \rightarrow \infty$.

Suppose we could observe an ensemble of $S$ starting values, $x_{1,1}, x_{1,2}, \ldots, x_{1, \mathrm{~S}}$, and again let successive observations equal the starting value to generate each of the $S$ resulting sequences, each of length $t$. The ensemble average as $S \rightarrow \infty$ will converge to the true mean, 0 .

In short, the time average for all $t$ is simply $x_{1}$, which is not equal to the ensemble average, which approaches 0 as $S \rightarrow \infty$.

Formally, the property known as ergodicity assures us that time averages converge to ensemble averages. In practice, one usually only has a single realization of the series, although it may be a very long series. By assuming ergodicity, one can use time averages to estimate ensemble averages. The idea behind ergodicity is that the process generating the data does not have any "sticky" values. That is, the process must not be trapable in a subset of its domain. Our example above was an extreme case for simplicity, in which the sequence became trapped at its starting value. In that case, unless we get to see repeated starting values, we cannot estimate the behavior of an ensemble of sequences generated from the same process.

A simple example of an ergodic process is a sequence of values $x_{1}, x_{2}, \ldots, x_{t}$, where each $x_{i}$ is an independent $N(0,1)$ observation. This again is a rather uninteresting time series, but it is obviously ergodic because the time average of any quantity of interest such as the mean or variance is guaranteed to converge to the corresponding ensemble average. 
In practice, ergodicity cannot be proven and must be assumed. The other essential property, stationarity, can be informally demonstrated. See Fig. 1c and Fig. 2a, where the original series has been differenced once and twice, respectively. By differencing, we construct a new series, $d_{t}=x_{t}-x_{t-1}$. The series of first differences will be shorter than the original series by one. The series of second differences will be shorter than the original series by two, and so on. The first differenced series shown in Fig. 1c appears to be stationary. However, the residuals from the regression of $x_{t}$ on $t$ appear to be a quadratic function of $t$. Also, the ACF of the residuals from the regression of $x_{t}$ decreases very slowly to zero as the lag increases. See Fig. 2c. The twicedifferenced series is shown in Fig. 1a, and the residuals from the regression of $x_{t}$ on $t$ and $t^{2}$ show a large ACF only at lag 1. See Fig. 2d. It appears that the twice-differenced series is stationary, so that the local dependency can be estimated. We will return to this example in Sec. 3.

Differencing is the most common method to remove a trend. If there is a linear trend, first differences suffice. If there is a quadratic trend, second differences suffice. A second-differenced series is obtained by differencing the first-differenced series. In general, for a $k$ th degree polynomial trend, $k$ th differencing suffices.

Of course, a trend can have a functional form other than a polynomial form so some transform other than differencing might be required. However, the point we wish to emphasize is that some transform of the data (often called a filter) must be used to construct a stationary series before the local properties can be estimated.

This report will mainly consider the analysis of stationary and ergodic time series. We therefore assume we can find some transform to remove any long-term trend.

Assume then that any long-term trend has been removed and consider the following autoregressive moving average (ARMA) model for a linear stationary time series $\left\{X_{1}, X_{2}, \ldots, X_{n}\right\}$.

The ARMA $(p, q)$ model is as follows:

$$
X_{t}=a_{o}+\sum_{j=1}^{p} a_{j} X_{t-j}+\sum_{j=0}^{q} b_{j} \varepsilon_{t-j}
$$

where the $a_{j}$ and $b_{j}$ are real constants, the $\varepsilon_{t-j}$ are independently and identically distributed (iid) $N\left(0, \sigma^{2}\right)$ random variables, and $t \in\{1,2, \ldots, n\}$. It is common to refer to $\varepsilon_{t-j}$ as the shock at time $t-j$, and the notation $N\left(0, \sigma^{2}\right)$ denotes the normal distribution with mean 0 and variance $\sigma^{2}$.

Note that $X_{t}$ is a linear combination of the past $p$ values of the series and of the past $q$ shocks. This class of ARMA models has been thoroughly studied and has become a prevalent tool for the analysis of time series, particularly since 1976 when Box and Jenkins developed a model-fitting strategy now referred to as Box-Jenkins methods. The ARMA modelling strategy is easily extended to nonstationary series by differencing the series to achieve stationarity. The extended modelling is called ARIMA modelling, where the acronym is auto-regressive, integrated moving average.

We will apply some of the Box-Jenkins methodology without explanation in Sec. 3. A good reference for the Box-Jenkins methodology is Chatfield's text. ${ }^{\text {' }}$ 
Part of the emphasis in this primer is to introduce methods that help decide if a nonlinear model is needed or whether a linear model will suffice. In view of the notation used in the linear ARMA model, we consider two possible ways to write a general nonlinear model. The nonlinear model should include the linear model as a special case.

\section{Model 1:}

State equation: $x_{t}=f\left(x_{t-1}, x_{t-2}, \ldots, x_{t-l}\right)$,

where both the function $f: \Re^{l} \rightarrow \Re^{1}$ and the lag $l$ are unknown. The function $f$ can be linear or nonlinear.

Assume we can only observe a corrupted version of $x_{t}$, say

Observation equation: $Y_{i}=x_{t}+\varepsilon_{t}$,

where the random error $\varepsilon_{t}=\varepsilon^{\prime}$, is a suitably defined function of $\varepsilon_{t}, \varepsilon_{t-1}, \ldots, \varepsilon_{t-q}$. The functional dependence of $\varepsilon^{\prime}$, on $\varepsilon_{t}, \varepsilon_{t-1}, \ldots, \varepsilon_{t-q}$ is linear for the ARMA model, but need not be linear for the more general nonlinear model. For ease of notation, Eq. (3) has been written using $\varepsilon_{t}$ rather than $\varepsilon^{\prime} t^{.}$

We have used a capital letter to denote that $Y_{t}$ is a random quantity. In contrast, according to Eq. (3), $x_{t}$ is not random, only unobservable.

Especially in signal processing it is common to seek an estimate of the unobservable state variable, $x_{t}$. Loosely speaking, the view is that we receive signal+noise and want to estimate the signal. We will not emphasize this viewpoint because our major goal is prediction. The ability to make good predictions is closely related to the safeguards goal of detecting anomalous data. A rather different model allows the function $f()$ to operate on the corrupted data as follows.

Model 2:

$$
X_{t}=f\left(X_{t-1}, X_{t-2}, \ldots, X_{t-l}\right)+\varepsilon_{t}
$$

Note that we use $X_{t}$ in Model 2 and $x_{t}$ in Model 1 . Models 1 and 2 are different because in Model 1 the observation error does not affect the dynamics of the map, $f()$, but in Model 2, $f()$ acts on the corrupted versions, $X_{t}$.

The ARMA model is a special case of Model 2, in which the function $f()$ is linear and the error $\varepsilon_{t}$ is a linear function of the present and past errors. As an aside, much recent research on specific nonlinear maps that generate the phenomenon known as chaos use Model 1. In fact, Model 2 is sufficiently different from Model 1 that many of the methods in use, such as estimating the fractal dimension, are much more difficult to apply with Model 2. See Ref. 2. Perhaps this is also a good place to mention that all models are wrong. After all, a model is an intentional simplification of a complex process. The test of our model will be how the prediction procedures, which we derive from the model, perform on particular data sets. In fact, although much research effort 
is spent in deriving optimal estimation procedures for particular time series models, often real data are best analyzed using the simplest techniques. One old standby is exponential smoothing, where the most recent few observations are used to predict the next observation. This is a common sense procedure that can be formally justified as the optimal method for particular ARIMA models. Such justification is largely academic because real-time series never obey a particular model exactly, and rarely even approximately obey a particular model for very long. Therefore, much of the research in time series is not useful for the practical analysis of time series. Having said that, we still intend to apply the more advanced tools developed to be optimal for the ARIMA model and tools that should be good for either of the two nonlinear models. We also apply exponential smoothing in one example. Naturally, we can showcase the tools on simulated data that actually do follow Model 1 or Model 2. To be fair, we will also apply the tools to real data. In Sec. 3 we describe how we will apply linear and nonlinear models to both linear and nonlinear time series.

\subsection{Linear and Nonlinear Modelling of Time Series}

Our main concern in Sec. 3 is modelling the local dependency of a time series. Therefore, any long-term trend must be removed, as discussed in Sec. 2.

Recall the ARMA Model and Models 1 and 2 in Sec. 3. The unknowns are the lag $l$, the functional form $f()$, and the error structure. The two major goals in Sec. 3 are to estimate $f()$ and to estimate $l$.

We can attempt to achieve these goals using either linear or nonlinear modelling.

\section{Linear Modelling of Time Series}

The major tool in the linear modelling of time series is the sample ACF as introduced in Eq. (1) of Sec. 2. Just as the ordinary correlation between any two random variables measures the degree of linear dependence, the ACF measures the degree of linear dependence between lagged values in a time series.

In linear modelling, we assume $f()$ is linear and that the error structure is linear. Using these assumptions, the Box-Jenkins methodology can be applied to estimate the linear coefficients that enable us to predict future values.

\section{Nonlinear Modelling of Time Series}

Our major tool in the nonlinear modelling of time series is the estimate, $\hat{M}\left(x_{1}, x_{2}, \ldots, x_{l}\right)$ of the conditional expected value,

$$
M\left(x_{1}, x_{2}, \cdots, x_{l}\right)=E\left(X_{t} \mid X_{t-1}=x_{1}, X_{t-2}=x_{2}, X_{t-l}=x_{l}\right) .
$$


Consider the lag = 1 case. A nonparametric estimate of the lag = 1 conditional mean is

$$
\hat{M}(x)=\left[\frac{1}{1-n} \sum_{j=1}^{n-1} X_{j+1} k\left(\frac{x-X_{j}}{h}\right)\right] /\left[\frac{1}{n} \sum_{j=1}^{n} k\left(\frac{x-X_{j}}{h}\right)\right],
$$

where $k$ is called the kernel. It is usually assumed that $k(x)$ evaluated at $x=0$ is the maximum value of $k$, and that $k(x)$ is a decreasing function of $|x|$. It is usually further assumed that $k$ is a symmetric probability density function such as the standard normal density. The parameter $h$ is the bandwidth, which determines the amount of smoothing. For more detail see Ref. 3, but the idea in Eq. (6) is straightforward. We have $n$ observations, $X_{1}, X_{2}, \ldots, X_{n}$, and seek an estimate of $X_{t+1}$ given the value $X_{t}=x$. The idea is to use all of the first $n-1$ observations, but weigh most heavily the observations that are nearest the value $x$. Suppose that $X_{10} \approx x$. Then we would heavily weigh the value of $X_{11}$ in the estimate of the conditional mean of $X_{t+1}$ given the value $X_{t}=x$. By use of a kernel $k(x)$, which has its maximum value at $x=0$, Eq. (6) weighs most heavily those observations $X_{t+1}$ that have $X_{t}$ close in value to $x$. Loosely speaking, if the bandwidth $h$ is very large then, relative to $h$, all observations are close in value to $x$, which is regarded as heavy smoothing. As an aside, a critical parameter in the performance of the conditional mean estimator given in Eq. (6) is the size of the smoothing parameter $h$. We have two versions of the conditional mean estimator. One version allows $h$ to vary according to the local density of the data. That version is called an adaptive method. In our experiments, we have had more success with the non-adaptive version, so we only report results for the non-adaptive version in this report. A nonparametric estimate of the conditional mean $M\left(x_{1}, x_{2}, \ldots, x_{l}\right)$ is also available if the lag $l>1$, but the idea is the same as the lag $l=1$ case, so we do not provide an equation here.

Reference 3 provides details about the conditions that must hold if the estimated conditional mean $\hat{M}(x)$ is to converge to the true conditional mean $M(x)$. Briefly, the time series must be stationary and geometrically ergodic. Also, there are smoothness requirements on the function $f()$, and the bandwidth $h(n)$ must satisfy the conditions that $h(n) \rightarrow 0$ and $n h(n) \rightarrow \infty$ as $n \rightarrow \infty$. In practice, the most critical issue is selecting $h(n)$. The most common approach is to experiment with a range of values for $h(n)$. In Eq. (6) we did not indicate that good values of $h$ depend on the value of $n$ because our intent was simply to introduce the idea of a nonlinear conditional mean estimator without getting involved in some of the finer details.

Whether we fit a linear or nonlinear model, the key check of a fitted model is the behavior of the prediction errors. If a model is successful, then prediction errors will contain no information about future prediction errors. In the case of linear modelling, there should be no correlation in the sequence of prediction errors. In the case of nonlinear modelling, the conditional expectation of a future prediction error should not depend on the present prediction error.

On the other hand, if prediction errors do contain information about future prediction errors, then inat information should be used to reduce the magnitude of future prediction errors. That is, the model should be revised. 
There are several techniques for estimating the lag $l$. For simulated data, there is likely to be a "true" lag, which we always hope to dis sover. For real data, we simply seek a good value to use for the lag.

For both linear and nonlinear models, the general idea in estimating the lag is that introducing a higher lag into the model can be justified only if the prediction error variance is suitably reduced. Because the prediction error variance is a nonincreasing function of the number of lagged variables used, an informal way to choose a lag is to plot the prediction error variance versus candidate lags. Accept the largest lag before the plot flattens out.

More formal ways are available. For linear models, we have used the Box-Jenkins methodology, in which certain lags are tried based on educated guesses using knowledge of the theoretical ACF for the particular ARMA model. The final lag is decided upon when prediction errors using that lag show no correlation, appear to have a constant variance, and do not depend on the predicted values themselves. For nonlinear models, we have used a cross validation technique as developed in Ref. 4.

And finally, because our major goal is prediction, we consider the mean (average) squared prediction error (MSEP) to be an important criterion. If two fitted models both pass all error diagnostics, we prefer the model with the lowest MSEP.

In the next section we consider some example time series.

\subsection{Examples of Linear and Nonlinear Modelling}

In this section we apply linear and nonlinear models to both linear and nonlinear time series. We analyze these linear time series:

1) $X_{t}=2 t^{2}-t+0.5 e_{t-1}+e_{t}$, where $e_{t} \sim N\left(0,500^{2}\right)$. This is the time series considered in Figs. 1 and 2. Because this time series is a quadratic function of time $t$, it could be considered a nonlinear time series. However, by analyzing its second differences, or by fitting $X_{t}$ to $t^{2}$ and $t$, it can be regarded as a linear time series.

2) $X_{t}=0.5 e_{t-1}+e_{t}$, where $e_{t} \sim N(0,1)$. This is a moving average, lag- 1 time series (MA(1)).

3) $X_{t}=0.5 X_{t-1}+e_{t}$, where $e_{t} \sim N(0,1)$. This is an autoregressive, lag-1 time series (AR(1)).

4) $X_{t}=0.5 X_{t-1}+0.5 e_{t-1}+e_{t}$, where $e_{t} \sim N(0,1)$. This is an autoregressive, moving average, lag-1 time series (ARMA $(1,1))$.

5) $X_{t}=0.5 X_{t-1}+0.5 e_{t-1}+e_{t}$, where $e_{t} \sim N(0,1)$. We observe $Y_{t}$, where $Y_{1}=X_{1}$, and $Y_{j}=X_{j-1}+X_{j}$ for $j \geq 2$. This is an integrated, autoregressive, moving average, lag-1 time series $(\operatorname{ARMA}(1,1,1))$.

We analyze these nonlinear time series:

6) $x_{t}=4 x_{t-1}\left(1-x_{t-1}\right)$, and we observe $Y_{t}=x_{t}+e_{t}$, where $e_{t} \sim N\left(0,0.1^{2}\right)$. This is the logistic map with an independent observational error structure. 
7) $x(j)=4 \sin \left(x_{j-1}\right)$ and we observe $Y_{t}=x_{t}+\cos \left(e_{t-1}\right)$, where $e_{t} \sim N\left(0,0.5^{2}\right)$. This is a nonlinear map with nonlinear observational error structure.

8) $X_{t}=-0.7 X_{t-1}+e_{t}$, if $X_{t-1} \leq 0$. Otherwise, $X_{t}=0.7 X_{t-1}+e_{t}$, where $e_{t} \sim N\left(0,0.5^{2}\right)$. This is a threshold AR model. See Ref. 5.

\section{Analysis of the eight examples}

We assume in this section that we must infer what process is generating the eight example time series. Because we know what is generating the simulated data, we can compare our inference to the true process.

In all cases, the linear modelling proceeds as follows:

A. Examine the time series for a trend. Experiment with the best way to remove the trend. Note that only the first example contains a trend.

B. Use the sample ACF of the detrended series to select an ARMA model, which includes selecting the lag. Selecting an ARMA model by this method relies on knowledge of the theoretical ACF of ARMA models. See Ref. 1 for further details.

C. Examine the residuals from the forecasted series. The forecasts are obtained by using estimated ARMA parameters. We divide each series into a training and a testing set. We use the training set to select a model and estimate its parameters. For all examples except the first example we use 200 training and 100 testing observations. We use fewer observations in the first example. Suppose that an MA(1) model is selected by analyzing the training set. Then, observation $i$ of the testing set is used to predict observation $i+1$ of the testing set.

D. If we use a lag-1 model, then 99 forecasts are made for the 100 testing observations, which leads to 99 residuals. If we use a lag- 1 model, then 98 forecasts are made, and so on. No matter what lag we use, the residuals must show no linear or nonlinear structure. If the residuals show no serial dependence, then the model is accepted as a candidate model.

E. A final model is selected from among all candidate models on the basis of parsimony (fewest parameters) and the MSEP of the forecast errors.

In all cases, the nonlinear modelling proceeds as follows:

A. Same as for linear modelling.

B. The sample ACF is not adequate for nonlinear time series. We use the conditional mean as described in Sec. 3. Implicit in the use of the estimated conditional mean is an estimate of the lag. We use a cross-validation scheme described in Ref. 4 and a plot of the MSEP versus the lag to estimate the lag. Having selected the lag, we use the conditional mean to predict future values. 
Steps C, D, and E are the same as for linear modelling. However, to monitor the residuals for dependence, we must consider both linear and nonlinear dependence.

We now analyze the eight example time series.

TIME SERIES 1: $X_{t}=2 t^{2}-t+0.5 e_{t-1}+e_{t}$, where $e_{t} \sim N(0,1)$.

\section{Linear Modelling Results}

Recall from Fig. 1 that either first or second differencing is needed to remove the trend for time series 1. Because of the quadratic trend in the residuals from the regression of $X_{t}$ on $t$ (Fig. 1d), we assume that second differencing is required. We have the choice of either analyzing second differences or analyzing the residuals from the fit of $X_{t}$ to $t^{2}$ and $t$.

Method (a). Analyze second differences. The true model for the second differences is the $\mathrm{MA}(3)$ model, $d 2_{t}=4+0.5 d 2_{t-3}-1.5 d 2_{t-1}+e_{t}$. The estimated model for the second differences was the MA(3) model, $d 2_{t}=3.6+0.45 d 2_{t-3}-1.3 d 2_{t-1}+e_{t}$.

The sample ACF of the residuals from the forecasts for lags 1 through 9 were within the error bounds of zero. However, because there are only 22 forecasts in the testing set, the error bounds were fairly large. The MSEP was 503884 . The theoretical MSEP is $500^{2}=250000$. Therefore, the MSEP is rather large.

Method (b). Analyze residuals from fitting $X_{t}$ to $t$ and to $t^{2}$. The true model is $\mathrm{E}\left(X_{t}\right)=$ $2 t^{2}-t$. The fitted model was $\mathrm{E}\left(X_{t}\right)=180.8+2.1 t^{2}-12.6 t$. The residuals had a large lag- $1 \mathrm{ACF}$ and a fairly large lag-3 ACF, suggesting either an MA(1) or an MA(3) model. A fitted MA(1) model had satisfactory residuals, showing negligible ACF. The MSEP was 402006 , about $20 \%$ less than the MSEP from method (a).

The MSEP of most interest is the MSEP in predicting the original series. So far, we have only considered the MSEP in predicting the residuals from a model fit in Method (a) to second differences and in Method (b) to residuals from a quadratic fit.

However, there is no difference in the MSEP in predicting the original series and the MSEP in predicting the errors in the forecasts of the original series. We illustrate the idea with Method (b); in Fig. 3a, the fitted quadratic from Method (b) is shown along with the original time series. We modify the fit by exploiting the estimated prediction error structure. That is, we use the estimated MA(1) coefficient of -0.54 (true value -0.5 ) to predict the residual for observation $t+1$ using the residual for observation $t$. In effect, we modify our next estimate using the error in the present estimate. The modified fit is shown in Fig. 3b. The resulting errors have an MSEP of 402006, which is the same as the MSEP in forecasting the errors from the quadratic fit.

We were puzzled why the MSEPs from both method (a) and method (b) were so much larger than the theoretical MSEP from this model. The larger MSEPs could have been caused by the short length of either the training or the testing sets. 
The short length of the training set implies that parameter estimates have large variances. The short length of the testing set implies that the forecast error variance is poorly estimated. The true forecast error variance should approach $500^{2}=250000$ as the training set and testing set get longer. Considering Method (b), we fit an MA(1) model to errors from the true quadratic function $X_{t}+2 t^{2}-t$. The resulting MSEP was 388581 . Recall that the MSEP for the fitted model was 402006. Therefore, the short length of the testing set used to estimate parameters had somewhat of an impact. The other important fact is that the MSEP is estimated using only the length-25 test series. If we simply fit the quadratic model to $X_{t}$ and ignore the fact that the resulting errors are correlated, the MSEP of the forecast error in the length-100 time series, which included both the training and testing sets, is 367541 . However, the MSEP of the forecast error in the length-25 testing set is 442459 . By accounting for the correlation between successive errors, we expect to reduce the forecast error by about $25 \%$, to about 331844 . However, recall that the MSEP was 402006 , so we did not achieve the $25 \%$ reduction. As an aside, the $25 \%$ reduction is expected because of the particular coefficient $(-0.5)$ in the MA(1) model.

In summary, the explanation for our larger-than-expected MSEP is that the training and testing series were both rather short. Because there were only 75 training observations, the parameter estimates were noticeably incorrect. And, because the testing set had only 25 observations, we happened to overestimate the true MSEP. Refer again to Fig. 3. Note that the last 14 forecasts in Fig. 3a are all larger than the observed, so the last 14 forecast errors are negative. There is improvement in Fig. 3b. Only the last 12 forecast errors are negative. The 24 forecast errors for Fig. 3b are shown in Fig. 4a. The sample ACFs of these 24 forecast erors are shown in Fig. $4 \mathrm{~b}$. There is nothing in Fig. 4a or $4 \mathrm{~b}$ to suggest that the model could be improved. That is, the errors show no serial correlation. The testing series simply happened to be systematically overestimated by the quadratic fit in the last part of the series. If we repeatedly generated data from this model, we would have observed the forecast error MSEP vary randomly around its expected value of 250000 .

\section{Nonlinear Modelling Results}

Because the error structure is linear, the results from nonlinear modelling of this time series are expected to be worse than the results from linear modelling. Nonlinear modelling as we have defined it, is not incorrect for a linear time series. It is merely inefficient. The reason is that the linear ARMA model is a special case of our nonlinear model. However, if the true model is linear, the training set is used more efficiently if we assume linearity and estimate the linear coefficients. In general, we have found that provided the training set contains at least about 100 observations, there is negligible difference between using our nonlinear estimated conditional mean and using linear modelling. The estimated conditional mean will be linear if the true model is linear.

Our approach was to use the fitted quadratic we obtained from the linear modelling and apply the conditional mean estimator to the residuals. By using a lag-1 estimated conditional mean, we reduced the MSEP from 442459 to 419673 . The forecast errors showed no correlation. 
Recall that, using the linear modelling, we reduced the MSEP from 442459 to 402006 , so linear modelling is the preferred method. Had we used a longer series, the nonlinear model's MSEP would be closer to the linear model's MSEP.

Time series 2-8 will each have 300 observations. For estimation purposes, we divide the 300 observations into 200 training observations and 100 testing observations.

TIME SERIES 2: $X_{t}=0.5 e_{t-1}+e_{t}$, where $e_{t} \sim N(0,1)$.

\section{Linear Modelling Results}

Time series 2 is plotted in Fig. 5a. Before we present the results for time series 2, note that the conditional variance of $X_{t}$ conditioned on $X_{t-1}$ and on $e_{t-1}$ is 1.0. That is, if we are given the values of $X_{t-1}$ and $e_{t-1}$, then we can predict $X_{t}$ as $\hat{X}_{t}=0.5 e_{t-1}$. The variance of the prediction error will be the variance of $e_{t}$, which equals one. Also note that it is redundant to condition $X_{t}$ on $X_{t-1}$ and on $e_{t-1}$. We need only condition on $e_{t-1}$. If we only condition $X_{t}$ on $X_{t-1}$, then it can be shown that the true variance of the prediction error will be $1.25\left(1-0.4^{2}\right)=1.05$, where 0.4 is the correlation between $X_{t}$ and $X_{t-1}$. Therefore, it is better to condition $X_{t}$ on $e_{t-1}$. Of course, the errors $e_{t}$ are not actually observed, so they must be estimated. We refer the reader to Chatfield's text ${ }^{1}$ for more detail about making forecasts for ARIMA models using Box-Jenkins methods.

In the Box-Jenkins methodology, the correct ARMA model will be selected for time series 2-5 if the training series is sufficiently long. Time series 2-5 use 200 training observations, which is sufficiently long to almost always select the correct ARMA model. We are practically guaranteed that the sample ACF will suggest the correct lag and the correct ARMA model when the true model is an ARMA model and the training series contains as many as 200 observations. The only other concern is how well the ARMA coefficients will be estimated. Again, 200 observations is sufficient to obtain an accurate estimate of the ARMA coefficients.

We therefore expect that the forecast error MSEP will be approximately 1.0 for time series 2 , and that the estimated MA(1) coefficient will be approximately 0.5 .

The 100 testing observations had a variance of 1.29 . The theoretical variance is 1.25 for an infinitely long series. The estimated MA(1) coefficient was 0.48 and the residuals from the fitted MA model had a variance of 1.01 .

As stated above, we anticipated an excellent performance by the fitted ARMA model. That is, we expected to select the correct ARMA model, which was MA(1), and we expected the estimate of the MA(1) coefficient to be close to its true value of -0.5 . Also, for the remainder of our examples, unless stated otherwise, assume that the diagnostics for the forecast errors were acceptable. That is, there are no unusual patterns in the forecast errors, and there is no apparent relation such as a large ACF between the forecast errors that should be modeled. Performing diagnostic checks of the forecast errors is important, but we need not present the diagnostic plots each time. 


\section{Nonlinear Modelling Results}

Our nonlinear lag-selection methods selected a lag-1 model. However, the lag estimate was in doubt because there was not unanimous agreement on the correct lag. Lag estimation using our nonlinear autoregressive modelling strategy is expected to be troublesome in MA models. We will discuss this issue momentarily.

The residuals from the conditional mean forecasts had a variance of 1.11 . Recall from the first paragraph in the linear modelling results that we expect a forecast error MSEP of about 1.05 if we only condition $X_{t}$ on $X_{t-1}$. Stated another way, suppose we use the estimated correlation between $X_{t}$ and $X_{t-1}$ to predict $X_{t}$ from $X_{t-1}$. That is, if we assumed a linear relationship between $X_{t}$ and $X_{t-1}$ but did not use the full power of the Box-Jenkins forecasting methodology, we would expect a forecast error MSEP of about 1.05 rather than 1.0. We experimented with this idea, and we did obtain a forecast error MSEP of about 1.05 in the testing set.

We mention this here because the idea can be applied to improve our nonlinear prediction methodology. If the forecast errors from an estimated conditional mean show any structure (linear or nonlinear), we can exploit this structure to improve our nonlinear prediction. In the present example, the errors from the estimated conditional mean approach were correlated, so we suspect that the errors have a linear structure. We could use linear modelling to exploit and then eliminate this linear structure, or continue to use the more general nonlinear conditional mean approach to do the same thing. Here is the idea in more detail for the nonlinear conditional mean approach:

The forecast error $e_{t-1}$ appears to be related to the next forecast error $e_{t}$. We can use the present forecast error to predict the next forecast error.

We therefore apply the conditional mean estimation procedure twice. First, we apply it to estimate $X_{t}$ from $X_{t-1}$. From this estimation, we obtain 199 residuals in the length-200 training series. Upon detecting the correlation (or nonlinear structure) in the prediction errors from the training series, we apply the conditional mean estimation procedure a second time but this time to the errors in the training set. We then modify our first estimate of $X_{t}$, which used only $X_{t-1}$, by also using the observed error in estimating $X_{t-1}$ from $X_{t-2}$ to predict the next error. The final estimate of $X_{t}$ is the sum of the first estimate and the expected value of $e_{t}$ given the value of $e_{t-1}$.

We applied this two-step conditional mean estimator to the MA(1) example. The resulting forecast error MSEP reduced from 1.11 (one-step procedure) to 1.02 (two-step procedure).

Of course, we had to work a little harder than we did with the Box-Jenkins methodology, but we achieved nearly the same forecast error MSEP. This is a testimony to the claim that little is lost by not assuming a linear model when the true model is linear, provided the series is sufficiently long. However, it should be noted that our nonlinear estimation method is designed for autoregressive models rather than moving average models. Therefore, an ad hoc procedure such as the above two-step procedure will be needed to handle MA models. We hope to replace our ad hoc procedure with a general strategy that can treat MA models as well as ARMA models. We also mention here that even though, for example, an MA(1) model can be expressed as an infinite order AR model, we would not ordinarily attempt to fit an infinite order AR model because a simple MA(1) model would be preferred. However, we have noticed a tendency for our lag estimation procedures to 
estimate a lag of two to four when the correct model is MA(1), having lag 1 . We can understand the source of this error by writing the MA(1) as an infinite order AR model.

TIME SERIES 3: $X_{t}=0.5 X_{t-1}+e_{t}$, where $e_{t} \sim N(0,1)$.

\section{Linear Modelling Results}

Time series 3 is plotted in Fig. 5b. The estimated AR(1) coefficient was 0.52 , and the variance of the testing series was 1.28. The true $A R(1)$ coefficient is 0.5 and the theoretical variance is 1.33 .

The conditional variance of $X_{t}$ conditioned on $X_{t-1}$ is 1.0 . The variance of the forecast errors in the testing set was 1.01 .

As we anticipated, there was no difficulty in identifying the correct ARIMA model, and the estimated AR(1) coefficient was very close to the true AR(1) coefficient.

\section{Nonlinear Modelling Results}

Our nonlinear lag estimation procedures estimated lag 1. The variance of the forecast errors in the testing set was 1.01 , and the forecast errors passed the diagnostic tests.

Therefore, the AR(1) model was equally well modeled using linear or nonlinear methods. Recall that the nonlinear conditional mean method was a little awkward to apply to the MA(1) model. The reason is that in the MA(1) model to predict $X_{t}$, we must condition on the previous error rather than simply on the previous observation, $X_{t-1}$.

TIME SERIES 4: $X_{t}=0.5 X_{t-1}+0.5 e_{t-1}+e_{t}$, where $e_{t} \sim N(0,1)$.

\section{Linear Modelling Results}

Time series 4 is plotted in Fig. 6a. The estimated AR(1) coefficient was 0.49 , and the estimated MA(1) coefficient was -0.55 . The true AR(1) coefficient is 0.5 and the true MA(1) coefficient is -0.5 . The theoretical variance of $X_{t}$ is 2.0 . The variance of the testing set was 2.21 .

The conditional variance of $X_{t}$ conditioned on $X_{t-1}$ is 1.0 . The variance of the forecast errors in the testing set was 1.01 .

As we anticipated, there was no difficulty in identifying the correct ARIMA model, and the estimated $A R(1)$ and MA(1) coefficients were very close to the true $A R(1)$ and MA(1) coefficients.

\section{Nonlinear Modelling Results}

Our nonlinear lag estimation procedures estimated lag 1. Because of the presence of the MA(1) term, we had to perform the two-step procedure as described in the MA(1) example. Upon completion of the two-step procedure, the variance of the forecast errors in the testing set was 1.02 , and the forecast errors passed the diagnostic tests.

Therefore, the ARMA $(1,1)$ model was equally well modeled using linear or nonlinear methods. 
TIME SERIES 5: $d 1_{t}=0.5 d 1_{t-1}+0.5 e_{t-1}+e_{t}$, where $e_{t} \sim N(0,1)$ and $d 1_{t}=X_{t}-X_{t-1}$.

\section{Linear Modelling Results}

Time series 5 (not differenced) is plotted in Fig. $6 \mathrm{~b}$. Notice that the nonstationarity of $X_{t}$ is evident in Fig. $6 \mathrm{~b}$ to the trained eye. Review the time series plots of time series 2-5 in Figs. 5 and 6. Time series 5 looks very different from time series $2-4$. With experience, it is possible to recognize that a series appears to have a slowly changing mean. We know that time series 5 is not stationary, but that its first differences are stationary.

We anticipated that the sample ACF of the training set would help confirm that the original series was nonstationary and hence needed to be differenced. In Fig. 7a, we show the time series plot of the first differences of time series 5 . Notice that this plot looks identical to the plot of time series 4. In fact, it is identical because we used the same random number seed to generate all of our series. In Figs. $7 \mathrm{~b}$ and $7 \mathrm{c}$ we show the plot of the sample ACF of the training set for the original series and the differenced series, respectively. It is clear from the extremely slowly decaying ACF in Fig. $7 \mathrm{~b}$ that the original series is not stationary. The ACF of the first differences decays to zero at a rate that reflects the AR(1) dependence. The true ACF of an AR(1) model with a positive AR(1) coefficient decays exponentially to zero.

We analyze the differenced series, $d 1_{t}$. It is a simple matter to transfer predictions of the differenced series to predictions of the original series.

For $d 1_{l}$, the estimated AR(1) coefficient was 0.49 , and the estimated MA(1) coefficient was -0.55 . The variance of the testing series was 1.28 . The true $\operatorname{AR}(1)$ coefficient is 0.5 and the true $\mathrm{MA}(1)$ coefficient is -0.5 . The theoretical variance of $d 1_{t}$ is 2.0 . The variance of the testing set was 2.21 .

The conditional variance of $d 1_{t}$ conditioned on $d 1_{t-1}$ is 1.0 . The variance of the forecast errors in the testing set was 1.01 .

As we anticipated, there was no difficulty in identifying the correct ARIMA model, and the estimated AR(1) and MA(1) coefficients were very close to the true AR(1) and MA(1) coefficients.

\section{Nonlinear Modelling Results}

As we explained above, there is no difficulty in applying nonlinear modelling to the differenced series. We obtained the same results as we did for time series 4 .

This concludes the analyses of the five examples of linear time series. All five series were considered to be linear. We could have considered time series 1 to be nonlinear because it involved a quadratic dependence on time. However, its second differences are linear, as are its residuals from a quadratic fit.

We now consider the three nonlinear time series.

TIME SERIES 6: $x_{t}=4 x_{t-1}\left(1-x_{t-1}\right)$, and we observe $Y_{t}=x_{t}+e_{t}$, where $e_{t} \sim N\left(0,0.1^{2}\right)$. Assume $x_{0} \in(0,1)$. 
Time series 6 is known as the logistic map with observational error. Notice that if the map $f(x)=4 x(1-x)$ were applied to the noisy variable, $Y_{t}$, then the map is likely to diverge to $-\infty$.

In this section we introduce another plot for studying the lag-1 behavior of a time series. It is a simple scatterplot of $Y_{t}$ versus $Y_{t-1}$.

In Fig. 8a we show a scatterplot of the logistic map observed with no error. If the observational error variance is too large, we will fail to detect the quadratic structure. In Figs. $8 \mathrm{~b}$ and $8 \mathrm{c}$ we show the logistic map observed with $e_{t} \sim N\left(0,0.1^{2}\right)$ and $e_{t} \sim N\left(0,1^{2}\right)$, respectively. We analyze the series shown in Fig. 8b, in which the quadratic structure is visible.

\section{Linear Modelling Results}

The time series plot of the training set is shown in Fig. 9a and its sample ACF is shown in Fig. 9b. Because we detect negligible ACF in the original series, we cannot directly apply linear modelling. Notice that the quadratic dependence of $Y_{t}$ on $Y_{t-1}$ is such that the correlation between $Y_{t}$ and $Y_{t-1}$ is both positive and negative over the range of $Y_{t}$. We might have, therefore, anticipated to detect negligible $\mathrm{ACF}$.

However, we can still attempt to apply linear modelling to $Y_{t}$, similarly to the way we applied linear modelling to time series 1. Due to the apparent quadratic structure, we regress $Y_{t}$ on $Y_{t-1}$ and $Y_{t-1}^{2}$ and analyze the residuals. Based on Fig. $8 \mathrm{~b}$, we expect that a zero intercept regression model is appropriate. We experimented with both a zero and non-zero intercept. There was negligible difference in the forecast errors. We report the non-zero intercept case.

See Figs. 10 and 11. A successful linear model was fitted by regressing $Y_{t}$ on $Y_{t-1}$ and $Y_{t-1}^{2}$. In Fig. 10 we compare the observed to the predicted values for the first 50 observations in the testing set. Figure 11a is a time series plot of the forecast errors for observations numbered 202 through 300 in the testing set. Figure $11 \mathrm{~b}$ confirms that the forecast errors in Fig. 11a are not correlated.

Because the residuals showed no structure, no further modelling was required. The forecast error MSEP for the testing series was 0.07 . As a reference, the variance of the testing series is 0.14 . This means that if we simply used the mean of the training series to predict each value in the testing set (a constant predictor), the MSEP would be essentially 0.14 . If we include the correction due to the testing and training set having slightly different means, the MSEP would be approximately 0.15 . We have not successfully calculated the theoretically smallest possible MSEP. Of course, a lower bound for the smallest possible MSEP is 0.01 because that is the variance of the observational error. At any rate, the forecast error MSEP of 0.07 for the linear model is acceptably small.

\section{Nonlinear Modelling Results}

In applying the nonlinear conditional mean estimator, it is not necessary to first regress $Y_{t}$ on $Y_{t-1}$ and $Y_{t-1}^{2}$. Recall from Fig. $8 \mathrm{~b}$ that the quadratic dependence of $Y_{t}$ on $Y_{t-1}$ is apparent, and the conditional mean estimator can capture this fact.

In Fig. 12a we show the predicted values using our conditional mean estimator superimposed on the true values of a scatterplot of $Y_{t}$ versus $Y_{t-1}$ for the testing series. In Fig. $12 \mathrm{~b}$ we show the 
predicted values using our conditional mean estimator superimposed on the true values of observations numbered 202 through 300 in the testing series. Figure $12 \mathrm{~b}$ should be compared to Fig. 10 .

The MSEP of the forecast errors was 0.07 , which is the same result we had for the linear model. However, our procedure for estimating the lag suggested that lag 2 is preferable to lag 1. Also, the sample ACF of the forecast errors had a lag-1 ACF of 0.23 . By taking advantage of this $\mathrm{ACF}$ at lag 1, we could follow the two-step procedure described for time series 2 (use the present forecast error to predict the next forecast error, and thereby modify the next prediction) to reduce the MSEP of the forecast errors to 0.06 . This is a modest reduction, but we prefer not to accept a model with a large lag-1 ACF in the forecast errors, so we recommend the two-step procedure. Alternatively, we could have fit a lag- 2 model from the beginning.

In future research, we plan to calculate the theoretically smallest possible MSEP for this model. At present, the linear and the nonlinear models perform similarly.

TIME SERIES 7: $x(j)=4 \sin \left(x_{j-1}\right)$ and we observe $Y_{t}=x_{t}+\cos \left(e_{t-1}\right)$, where $e_{t} \sim N\left(0,0.5^{2}\right)$. Th is is a nonlinear map with nonlinear observational error structure.

Time series 6 was a nonlinear map with !inear observational error. There was only a small advantage in using a nonlinear model rather than a linear model. Recall that in our linear model we regressed $Y_{t}$ on $Y_{t-1}^{2}$ and analyzed the residuals for linear structure. This kind of approach has been heavily used in statistics. If we want to apply simple linear regression to a nonlinear functional relationship, we first seek a transform to approximate linearity. We also were able to treat time series 1 as a linear time series by first removing the quadratic trend.

Time series 7 is a nonlinear map with nonlinear observational error structure. Figure 13a is a time series plot of time series 7. Figure $13 \mathrm{~b}$ is a scatterplot of $Y_{t}$ versus $Y_{t-1}$, which reveals the periodic dependence of $Y_{t}$ on $Y_{t-1}$.

\section{Linear Modelling Results}

The sample ACF of the training set is shown in Fig. 14a. The periodicity of the sample ACF is evident. Again we can attempt to discover a reasonable transform to apply linear modelling. Suppose we are observant enough to notice that it would be reasonable to regress $Y_{t}$ on $\sin \left(Y_{t-1}\right)$ in the testing set. The resulting fit is $Y_{t}=-0.0165+2.76 \sin \left(Y_{t-1}\right)$. The forecast errors using this fit in the testing set are shown in Fig. 14b, and their ACF is shown in Fig. 14c. The forecast error MSEP in the testing set is 4.51 , whereas the variance of the testing set is 8.13 . Therefore, the regression of $Y_{t}$ on $\sin \left(Y_{t-1}\right)$ is useful for providing a reasonable forecasting method. However, from Fig. 14c, we see that the forecast errors are not independent, so the model obtained by regressing $Y_{t}$ on $\sin \left(Y_{t-1}\right)$ is not complete. We attempted to fit an MA(1) model to the forecast errors because of the large lag-1 ACF, but the fitted model's residuals still were not independent. Rather than pursue linear modelling further, because we know there is nonlinear structure in the errors, we turn to nonlinear modelling. 


\section{Nonlinear Modelling Results}

The estimates of the lag were inconclusive, suggesting either lag 1 or lag 2. Therefore, we tried both lag 1 and lag 2. The lag-1 model had a forecast error MSEP of 4.81. The lag-2 model had a forecast error MSEP of 3.75. The sample ACF of the lag- 1 and lag- 2 model errors are shown in Figs. 15a and 15b, respectively. It is evident from Fig. 15a that forecast errors from the lag-1 model still exhibit periodicity. There is negligible periodicity in the lag-2 model. Unfortunately, the true model is a lag-1 model. We therefore tried the two-step method as described in the MA(1) time series to remove the dependence in the forecast errors for the lag-1 model. Using the two-step procedure, we reduced the MSEP from 4.81 to 4.69 , but the ACF of the forecast errors was suspicious (approximately -0.2 ) at lags 3 and 4 . This was disappointing because the correct model is a lag-1 model, but we prefer the lag-2 model on the basis of the MSEP and forecast error diagnostics.

Concerning the forecast error MSEP, it is not surprising that, for the linear regression of $Y_{t}$ on $\sin \left(Y_{t-1}\right)$, it had a slightly lower MSEP than the nonlinear lag-1 model. After all, regression of $Y_{t}$ on $\sin \left(Y_{t-1}\right)$ was the best choice we could have made. However, for general nonlinear functions, we cannot expect to find such an effective transform that linear modelling can be used.

In conclusion, we were not able to decompose the nonlinearity of time series 7 into the nonlinearity of the $x_{t}$ as a function of $x_{t-1}$ and the nonlinearity of the lag-1 noise. In fact, we would probably accept a lag-2 model even though the true model is lag 1 . Therefore, neither the linear nor the nonlinear modelling was completely satisfactory for time series 7. Part of the difficulty is the cosine dependence on the lag- 1 error. Recall that $\cos (x) \approx 1$ for small $x$, whether $x$ is positive or negative. It would be interesting to derive the theoretically lowest possible forecast error MSEP for this model.

TIME SERIES 8: $X_{t}=-0.7 X_{t-1}+e_{t}$, if $X_{t-1} \leq 0$. Otherwise, $X_{t}=0.7 X_{t-1}+e_{t}$, where $e_{t} \sim N\left(0,0.5^{2}\right)$. This is a threshold AR model. See Ref. 2 .

\section{Linear Modelling Results}

Figure 16 shows a scatterplot of $X_{t}$ versus $X_{t-1}$, with a nonlinear fit superimposed. We can see from the superimposed curve that there is some form of nonlinearity operating. We could attempt to fit separate linear regressions for positive and negative values of $X_{t-1}$ because the nonlinear fit suggests that two linear regressions would suffice. If instead we study the ACF of the training series, we would attempt to fit an AR(1) model. We did so, and the AR(1) coefficient was estimated to be 0.62 . However, the forecast errors from the fitted AR(1) model fail the usual diagnostics, and the forecast error MSEP of the testing set is the same as the variance of the testing set. Therefore, we might as well have not even fitted a model. It is easy to understand why a simple AR(1) model will not perform well. The lag-1 coefficient changes sign depending on the value of $X_{t-1}$.

Because our nonlinear conditional mean estimator works by using local linear modelling (all functions are linear over a short enough range), our nonlinear approach will essentially result in two linear regions. We therefore do not attempt to fit separate linear regressions for positive and negative values of $X_{t-1}$. 


\section{Nonlinear Modelling Results}

We obtained very good results with our nonlinear conditional mean estimator. The forecast errors passed the diagnostic tests, the fnrecast error MSEP was 0.26 , and the theoretically lowest achievable MSEP is 0.25 . Recall that the AR(1) model (time series 3 ) was also very easily handled by our nonlinear conditional mean estimator. The two-step procedure is not needed because there is no need to know the value of the previous error in making the next forecast.

In Fig. 17a we show observations numbered 202 through 250, which are the first 49 observations in the testing set that we attempt to predict. Also shown in Fig. 17 are the corresponding 49 predicted values. There is a curious pattern in Fig. 17a. If we translate the predicted values back by one time unit, we obtain Fig. 17b. We included Fig. 17b because it illustrates how our nonlinear forecasting procedure is operating in this example. The forecast of the next observation is a fraction of the present observation. That is, we shrink the present observation toward zero in absolute value to estimate the next value. Note that negative observations are also reflected about zero, as they should be. We included the horizontal line at $X_{t}=0$ to help see the reflection of negative values about zero. Remember that we are plotting the prediction of the next observation in Fig. 17b.

In summary, the nonlinear model was successful for time series 8 . Also, we have seen again that a linear model could be successful for time series 8 . However, it is easier to simply let our nonlinear conditional mean estimator do the work for us, especially if there are several local linear models.

This concludes the analysis of the eight examples of simulated time series. The major points we wish to emphasize are as follows:

1. It is important to divide the analysis of time series into local and trend analyses. Any longterm trend will conceal local dependencies.

2. Because we adopted a model fitting and testing approach, we always perform diagnostic tests of a fitted model by analyzing the residuals from that model. The residuals should exhibit no pattern, no serial dependence, and have a stationary variance. Loosely speaking, if the residuals do not appear to be independent and identically distributed over time, then the structure in the residuals should be exploited to modify the model.

3. For fairly long time series, there is little lost in terms of the MSEP by not assuming a linear model when the true model is linear. Of course, if the true model is linear, we would like to know that to better understand the process generating the data. In practice, we suggest performing a test for linearity and then using either linear or nonlinear modelling, as appropriate. We do not discuss formal tests for linearity in this report. See Ref. 5 for further details. An informal test for linearity is simple and effective: plot the estimated conditional mean from our nonlinear conditional mean estimator. It will look linear if the true model is linear, and it will look nonlinear if the true model is nonlinear. 
4. The five examples of linear time series exactly followed particular ARMA models. We therefore expected and demonstrated great success from the Box-Jenkins methodology. The Box-Jenkins methodology is straightforward, but somewhat involved. It is often the case that a simpler method such as exponential smoothing (use a weighted average of the most recent observations to predict the next observation) works as well as any method on real data. For example, attempts to fit an ARIMA model to the daily closing price of an individual stock on the New York Stock Exchange usually suggest that a nonstationary (moving mean) model is appropriate. Therefore, the best forecasting method is some type of moving average, which weighs the most recent observations most heavily.

5. The three examples of nonlinear time series were best modeled by our nonlinear conditional mean estimator, but could also be modeled fairly well with clever use of linear modelling. In time series 6 and 7, the true models were simple enough that a transform could be found before applying linear modelling. In time series 8 , two local linear models would suffice. For many real data sets, the functional form of the nonlinearity is not so simple, so we would not attempt linear modelling. It is easier to use our nonlinear conditional inean estimator.

In the next section we consider two real (not simulated) time series. We also mention two additional nonlinear modelling strategies.

\subsection{Analysis of Two Real Time Series}

In Sec. 5 we analyze two real time series. The first is probably best modeled with a simple MA(1) linear model. The second exhibits nonlinear properties and is therefore best modeled with our conditional mean estimator or with either of the three additional nonlinear modelling strategies that we introduce.

The first time series is 144 monthly material balances recorded over 12 years. A material balance (MB) is a term from nuclear safeguards that refers to the estimated amount of missing nuclear material, usually plutonium. Because nuclear material is measured with error, the question of whether any material is missing is a statistical question. We approach this problem making no assumptions about the process generating the data.

In Fig. 18a we show the time series plot of the 144 monthly MBs. We denote the observed MB at time $t$ by $X_{t}$. Notice that there are six large observations. Ignoring the possible serial dependence and assuming that an individual $\mathrm{MB}$ is approximately normally distributed, we expect about $5 \%$ of all MBs to lie outside plus or minus two standard deviations. Based on that rule, observation numbers $11,12,13,47,48,49,81,82$, and 83 are all suspiciously large. The plus and minus two standard deviation lines are shown on the time series plot. Note that the large observations come in pairs. This suggests that there is serial correlation in the time series. In Fig. $18 \mathrm{~b}$ we show the $\mathrm{ACF}$ of the series and observe the large lag-1 ACF, suggesting that an MA(1) is appropriate. 
Recall that we assume throughout this report that we have a training series that contains no outliers. That is, we require non-anomalous data to build our models that we intend for use in monitoring future data for anomalies. Therefore, we have misgivings about the effect that the six suspicious observations will have on our model building. We therefore trimmed the six large observations as follows. The sample correlation of the first 100 observations is -0.37 . To trim observation number 11, we computed the conditional expectation of $X_{11}$ given $X_{10}$, assuming a linear model. We then generated a pseudo-measurement for $X_{11}$ by generating a normal random variable having mean equal to the expected value of $X_{11}$ given $X_{10}$ and standard deviation equal to the standard deviation of the first 100 observations. We restrict attention to the first 100 observations because we chose those to be our training set. The last 44 observations will be our testing set.

The issue of how to identify and then treat outliers in the training set is a subject in itself. We have presented here the simplest possible way to avoid the issue so that we could assume that our training set contained no outliers.

\section{Linear Modelling Results}

The lag- 1 correlation in the trimmed data is -0.26 , so the trimming did reduce the magnitude of the estimated correlation. The ACF of the trimmed data still suggests that an MA(1) model is appropriate, and the scatterplot of $X_{t}$ versus $X_{t-1}$ did not reveal nonlinearity. As an aside, the presence of the six outlying observations caused the scatterplot of $X_{t}$ versus $X_{t-1}$ to appear nonlinear.

The fitted MA(1) model was acceptable with regard to all residual diagnostics and had an estimated MA(1) coefficient of 0.28 . (Our MA models are written so that a negative lag- 1 correlation implies a positive lag- 1 coefficient.) The MSEP of the 43 forecast errors in the testing set was 4212914 , which corresponds to a standard deviation of 2053. For comparison, the standard deviation of the testing set is 2218 , so there is something to be gained by fitting a simple MA(1) model to this data. This is only a modest reduction in the forecast error standard deviation, but the fact that an MA(1) model adequately describes this data provides information about how the MB sequence is being generated.

\section{Nonlinear Modelling Results}

We also applied our nonlinear conditional mean estimator to this data. Recall that the twostep procedure described for time series 2 in Sec. 4 is needed for MA(1) models. The resulting forecast errors were acceptable with regard to all residual diagnostics and had an MSEP of 4330545 , which corresponds to a standard deviation of 2081. Therefore, this time series is suitably well modeled using the two-step procedure with our nonlinear conditional mean estimator. However, because the linear fit was fine and had a lower MSEP, we prefer a linear model for this time series.

Concerning MB sequences in general, it is typical in the safeguarding of nuclear material such as plutoniur, 0 assume that $\mathrm{MB}$ sequences are jointly normally distributed. Periodic tests for loss of plutonium usually use conditional expectations based on the normality assumption. That is, the predicted value of the next observed $\mathrm{MB}$ is its expected value, conditioned on all previously 
observed values, which are correlated with the next observed MB. That procedure is well founded in statistical theory. However, if the process generating the MB sequence is well modeled by an MA(1) model, it is more efficient to exploit the Box-Jenkins methodology or our two-step conditional mean methodology because the previous forecast error can be used to help predict the next observation.

The second time series is monthly flour prices for the city of Buffalo, New York, from August 1972 to November 1980. The data are available in Ref. 7. In the area of nonproliferation of nuclear weapons, we anticipate that certain countries' activities will be monitored. For example, imports and exports of goods that are related to nuclear weapons technology as well as chemical emissions from the country will probably be monitored. The application we have in mind requires the assumption that the country is behaving in accordance with international agreements for a sufficiently long period in which we can generate training data. Recall that we have assumed throughout this report that our training data contains no anomalies. Presumably, certain exports and imports as well as chemical emissions will appear as anomalies if the country later begins a nuclear weapons program. The goal is to develop a forecasting method with the smallest possible MSEP so that an unusual observation is quickly detected. As always in statistical tests, we can make two types of errors: false alarms and failure to detect an anomaly. Reducing the MSEP helps reduce the probability of both types of errors.

In Fig. 19a we show three time series, corresponding to the monthly flour prices of Buffalo, Minneapolis, and Kansas City. We only model the prices for Buffalo but point out the potential for more involved modelling, using all three time series. Note that the flour prices of the three cities are strongly related. In Fig. 19b, we show the same three time series on a log scale. We prefer to analyze the original scale, but to most easily compare our results to the results in Ref. 6, we have used the log scale.

Admittedly, we do not anticipate that flour imports and exports will be one of the monitored goods, but the data were available to us and have already been analyzed with a method that allows us to introduce some other possible modelling strategies.

Our goal is to use the first 75 observations for training and the last 25 observations for testing.

\section{Linear Modelling Results}

The sample ACF of the log of the Buffalo flour prices was positive and very slowly decaying to zero, suggesting either a lag-1 or lag-2 AR model, or that the series should first be differenced because it might not be stationary. We were unsuccessful in fitting a model to the first differences because the sample ACF of the first differences looked like the ACF of a random, independent series. We were also initially unsuccessful in fitting either an AR(1) or an AR(2) model to the undifferenced series using the model estimation scheme in the statistical software package $S+$. Therefore, we tried another statistical software package designed specifically to model time series, developed to accompany a text on time series. The package is called ITSM: An Interactive Time Series Modelling Package for the PC. ITSM offers a good self-tutorial on modelling time series and can model vector observations. We have not investigated the numerical methods used to fit 
ARIMA models in the two packages S+ and ITSM, but the numerical method used by I'TSM did converge for the flour data. However, the estimated AR(1) coefficient was 1.02 and the estimated AR(2) coefficient was -0.045 . These coefficients barely satisfy the stationarity conditions for an AR model. ${ }^{1}$ We were surprised that the forecast error residuals passed the diagnostic tests and had an MSEP of 0.00166. This is a lower MSEP than reported for the same data in Ref. 7.

In some sense, the AR(2) model is satisfactory, but we must interpret with caution any model that is so close to being nonstationary. We were able to get estimated parameters in $\mathrm{S}+$ for the AR(1) case by choosing a starting value in the estimation scheme of 0.999 . The estimated AR(1) coefficient in an AR(1) model was 0.9999999. For an AR(1) model, stationarity requires the AR(1) coefficient to have an absolute value strictly less than 1 . We are therefore too close to nonstationarity for comfort! We were never able to get $S+$ to fit an AR(2) model and, even for the AR(1) model, the $\mathrm{S}+$ estimation method never did meet its convergence criterion, although forecasts were produced. As an aside, if we used a starting value for the estimation procedure of less than 0.999 , say 0.99 , the estimation procedure in S+ would not produce forecasts for the AR(1) model. And finally, the residuals from the fitted AR(1) model failed the diagnostic tests, although they did have a very small MSEP of 0.0017 .

The fitted AR(1) or AR(2) models are on shaky ground, although the forecast error MSEP from the AR(2) model was very small, even smaller than the MSEP in Ref. 7 which used va ious neural networks and obtained forecast errors ranging from 0.0034 to 0.0177 . Reference 7 used only the last 10 observations to estimate the MSEP, so part of the difference in performance could be explainable on that basis.

\section{Nonlinear Modelling Results}

Presumably, any time series that can be modelled with an AR model can be modelled with our nonlinear conditional mean estimator. Our estimates of the lag all suggested that lag 1 was appropriate.

The forecast errors from a nonlinear lag-1 model had an MSEP of 0.0097, which is considerably larger than the MSEP of the linear AR(2) model. However, the forecast errors had a positive and slowly decaying ACF, so we tried our two-step procedure using both lag-1 and lag-2 errors. The two-step procedure reduced the MSEP to 0.004 , but the forecast errors from the two-step procedure failed the diagnostic tests. Therefore, our nonlinear modelling could not be considered successful. We are puzzled by this and our only explanation is that the training series is too short for the nonlinear conditional mean estimator to perform well.

Recall from the end of Sec. 2 that in many real time series, the simplest techniques are the best. A possible explanation is that the process generating the data is not well characterized by a constant coefficient model. That is, there may be a simple model in effect at any instant, but the model is always changing.

Because we were not satisfied with either the linear or nonlinear models we tried, we decided to try the often-used technique known as exponential smoothing. Recall that the linear models suggested that the flour prices nearly follow a nonstationary random walk. That is, $X_{t}=X_{t-1}$, which is 
an AR(1) model with an AR coefficient equal to one. If in fact that is the true model, it can be shown that the optimal forecast is

$$
\hat{X}_{t}=(1-\alpha) \hat{X}_{t-1}+\alpha X_{t-1}
$$

where $\alpha$ determines the degree of smoothing. We used $\alpha=0.9$, which is a relatively large value to produce the forecasts shown in Fig. 20. These forecasts passed all diagnostic tests and had an MSEP of 0.0017 , which is the same as the MSEP from the more complicated AR(2) model. It is common to search for the value of $\alpha$ that minimizes the forecast error MSEP, but we were satisfied with the MSEP we obtained with our first trial value of $\alpha$.

We have seen that the linear modelling was adequate for the MB data and that no modelling is best for the flour data. Certainly there are data sets for which our nonlinear modelling is best. However, both the nonlinear and linear modelling strategies we have presented have limitations. For example, it would not be difficult to imagine a time series model such that observation $X_{t}$ had a complicated functional dependence on the past severai observations in the series and that the functional dependence actually changed form depending on either the values of the past several observations or on their trend or both. Our nonlinear modelling approach can still be used, but it would require long training series to discover the true functional form. Essentially, the nonlinear conditional mean approach can suffer from the "curse of dimensionality"; it would be nearly impossible to estimate the multidimensional function $f($ ) unless the series is very long. One recent proposal $^{11}$ attempts to restrict the function $f()$ to an additive function of the possibly transformed lagged values in the series.

It has also recently become popular to handle complicated maps $f()$ with neural networks. The flour data were analyzed in Ref. 6 using a neural network. Though it is probably better to simply use exponential smoothing for a single series, the emphasis in Ref. 6 was to model jointly the three series. It is then possible to exploit the relationships of the flour prices in the three cities at any particular month, as well as using previous values. Alternatively, one could pursue the modelling approach that is common in statistics, which is known VARMA (vector autoregressive moving average) modelling. VARMA modelling is restricted to linear modelling, whereas neural network modelling is more general. And neural network modelling is a special case of nonlinear modelling. There are several issues remaining to be studied in the application of neural networks to forecasting time series. Most notably, as in almost any application of neural networks, it is important to develop methods to assist in choosing the complexity of the network (for example, number of layers or the number of nodes per layer). How to best find the parameter values that yield a global minimum of the MSEP is also an important issue if the time series can cause search algorithms to get trapped in local minima. We are currently studying the advantages of using neural networks for forecasting time series. Our first attempts with time series $1 \mathrm{in} \mathrm{Sec.} 4$ produced less accurate forecasts than our linear and nonlinear methods. There are many possible neural networks, and we expect to discover one that does as well as our nonlinear conditional mean model for this time series. 
A less well known modelling strategy that is extremely promising is the application of the multivariate adaptive regression with splines methodology (MARS) ${ }^{8}$ A recent paper ${ }^{9}$ proposed the method, calling it ASTAR (adaptive spline threshold autoregression). It was successfully applied to a particular time series (yearly sunspot data) that has spawned many attempts to develop more elaborate modelling strategies. The ASTAR methodology is particularly well suited for the situation in which the functional dependence changes form depending on either the values of the past several observations or on their trend or both.

We have begun to experiment with the ASTAR method. For time series 1 in Sec. 4, we achieved an MSEP of 436353 without first differencing the series or fitting a quadratic. Recall that in Sec. 4 by simply fitting a quadratic to the series, we achieved an MSEP of 442459 , which was further reduced by exploiting the structure in the errors. The residuals from the ASTAR method had a fairly large lag- 2 and lag-3 ACF (approximately -0.2 and 0.2 , respectively). Therefore the first model we obtained from the ASTAR procedure needs to be refined.

Finally, we have written software to do the following three-step procedure for modelling a time series:

1) Divide the observations into intervals. For example, split the range of the training set into groups one and two, where group one is below the median of the training set and group two is above the median.

2) Search for a good lag, beginning with lag 1.

3) Search for a good number of divisions of the range of the training series. We illustrate the method using only two divisions: above and below the median.

To illustrate the search, first consider the lag-1 case. For each observation in group one, record the value of the next observation. Average these values. The average will be the forecast for any observation following an observation that is below the median. Do the same for each observation in group two. So far, the method is a crude version of our nonlinear conditional mean estimator, so can of fer no advantage in the lag- 1 case.

Now consider the lag- 2 case. Each pair $f()$, can be denoted as either $(1,1),(1,2),(2,1)$, or $(2,2)$ where, for example, if $X_{t}$ lies below the median and $X_{t+1}$ lies above the median, then denote $\left(X_{t}, X_{t+1}\right)$ as $(1,2)$. As in the lag-1 case, for each pair in the "bin" $(1,1)$, record the value of the next observation in the time series. Average these values. The average will be the forecast for any observation that follows a pair in the bin $(1,1)$.

We jointly search for a good lag and a good number of divisions of the range of the training series. Recall that we have emphasized the distinction between stationary and nonstationary time series. This method is not recommended for nonstationary time series because in a nonstationary time series what we observe in the training set is not necessarily a good indication of what we will observe in the testing set.

We have partitioned the observations into pairs (for lag 2), triples (for lag 3), and so on. The main motive is to model cycles. For example, if a large observation tends to follow the pair $(1,1)$, 
we will discover it with this method. We have just begun to experiment with this approach and have not yet had good results. This approach is actually available within the ASTAR methodology and is also implemented in a slightly different way in Ref. 10 . That is, to predict $X_{t}$, both ASTAR and Ref. 10 consider the pattern of the previous pair, triple, etc. We will refer to this approach as the bin approach. Because the ASTAR methodology is slower to apply, and often allows more generality than is needed, we wrote our own code to perform the bin approach.

The neural network approach, the ASTAR approach, and the bin approach all extend fairly easily to modelling vector observations recorded over time. This topic is beyond the scope of this report but is one subject of our current research.

\subsection{Summary}

The intent of this report has been to introduce the concept of forecasting time series for monitoring the series for anomalous data. In performing such a task, we inevitably make the usual two kinds of statistical error: false alarms and failures to detect anomalies. To minimize the rate of these errors, we seek forecasting methods with minimum forecast error MSEP.

We have introduced linear and nonlinear modelling and demonstrated their performance on simulated and real data. One important observation is that linear modelling will continue to be an important tool because transformations are often available to convert a nonlinear time series to a linear time series. If a linear model suffices, it is preferred because it is the simplest and it might provide subject-matter knowledge if a linear model is suitable.

We have avoided most of the technical detalls, except for the importance of model checking, especially diagnostic checks of the forecast errors. The forecast errors should exhibit no serial correlation and have a constant variance. Otherwise, the model is incomplete.

We have briefly mentioned three relatively recent modelling strategies: ASTAR, neural networks, and the bin approach. These three modelling strategies will be inferior to linear modelling if the time series is well approximated by an ARMA model. Recall that ARMA models are linear. To date, the ability of the more recent modelling strategies to outperform linear modelling has been evaluated case by case for particular nonlinear time series. Unfortunately, in the application of neural networks to the prediction of nonlinear time series, we often see no comparison between the performance of the network and a suitable linear model. In some cases, researchers fail to notice that a simple transform of the data can make linear modelling a good choice.

Finally, we have provided references to tests for linearity but, in the context of prediction, we can simply fit both linear and nonlinear models to a time series and choose the model with the smallest MSEP provided the errors pass the diagnostic tests. In nuclear safeguards and nonproliferation, this will provide a way of monitoring a time series for anomalous data. We can define an observation to be anomalous if it is larger than about twice the square root of the MSEP (based on the customary 0.05 false alarm probability and assuming approximate normality of the forecast errors). By choosing a model that minimizes the MSEP, this procedure will have the theoretically best detection probability for a given false alarm probability. 


\subsection{References}

1. C. Chatfield, The Analysis of Time Series: An Introduction, second ed. (Chapman and Hall, London, 1980).

2. Richard Smith, "Estimating Dimension in Noisy Chaotic Time Series," Journal of the Royal Statistical Society, Series B 54(2), 329-351 (1992).

3. Bjorn Auestad and Dag Tjostheim, "Identification of Nonlinear Time Series: First Order Characterization and Order Determination," Biometrika 77(4), 669-687 (1990).

4. B. Cheng and H. Tong, "On Consistent Nonparametric Order Determination and Chaos," Journal of the Royal Statistical Society, Series B 54(2), 427-449 (1992).

5. H. Tong, Nonlinear Time Series: A Dynamical System Approach (Clarendon Press, Oxford, 1990).

6. K. Chakraborty, K. Mehrotra, C. Mohan, and S. Ranka, "Forecasting the Behavior of Multivariate Time Series Using Neural Networks," Neural Networks 5, $961-970$ (1992).

7. G. Tiao and R.S. Tsay, "Model Specification in Multivariate Time Series," Journal of the Royal Statistical Society, Series B 51, 157-213 (1989).

8. J. Friedman, "Multivariate Adaptive Regression Splines," The Annals of Statistics 19(1), 1-141 (1991).

9. P. A. Lewis and J. G. Stevens, "Nonlinear Modeling of Time Series Using Multivariate Adaptive Regression Splines (MARS)," JASA 86(416), 864-877 (1991).

10. A. Zardecki, "Tests of Improved Pattern Recognition Methods," Los Alamos National Laboratory, Safeguards Systems Group report N-4/93-655 (1993).

11. R. Chen and R. Tsay, "Nonlinear Additive ARX Models," JASA 88(423), 955-966 (1993). 


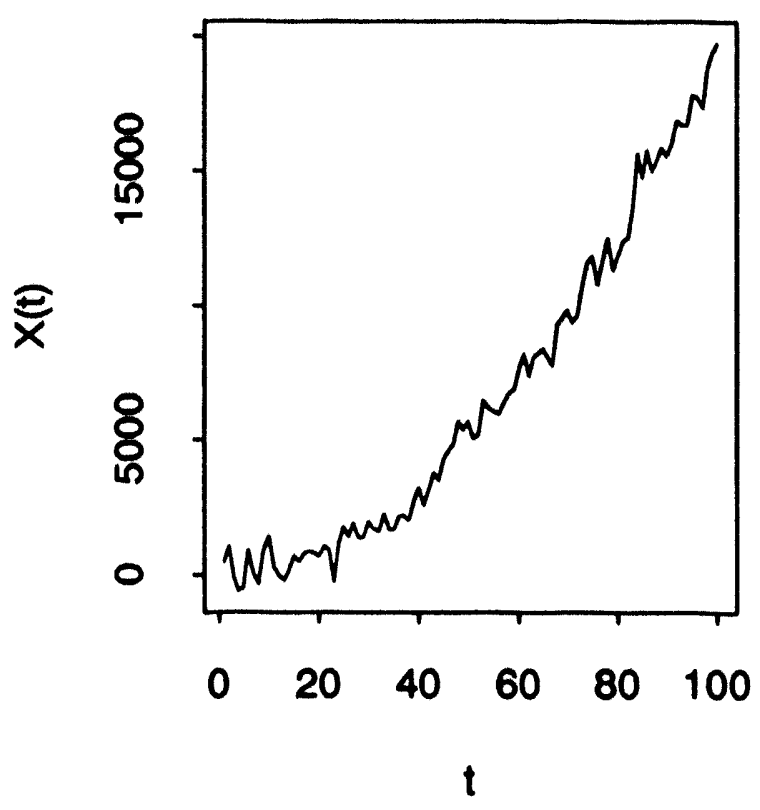

Fig. Ia. $X(t)$ versus $t$.

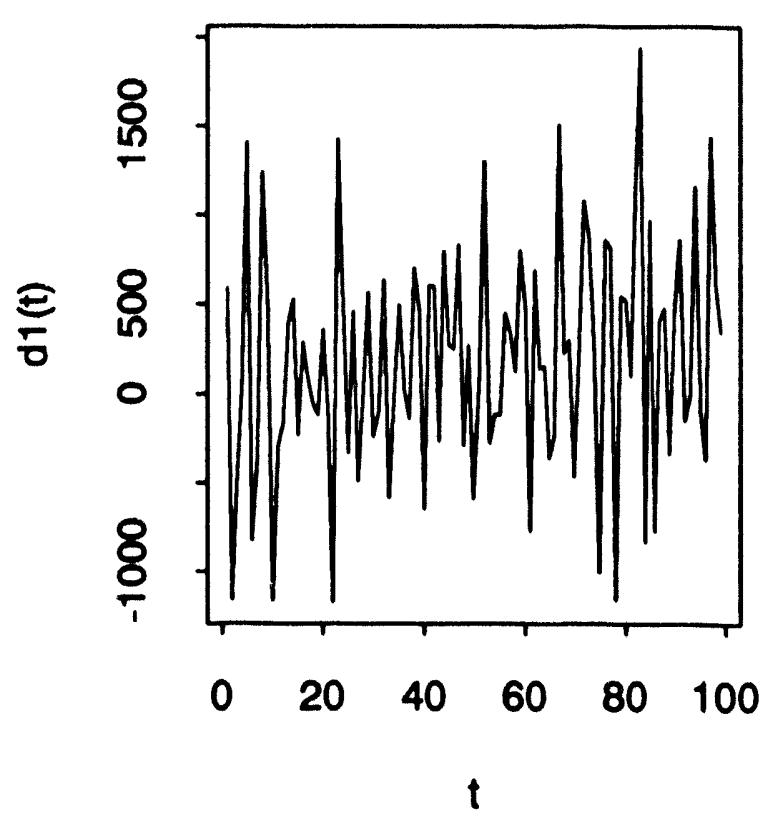

Fig. 1c. $d 1(t)=X(t)-X(t-1)$ versus $t$.

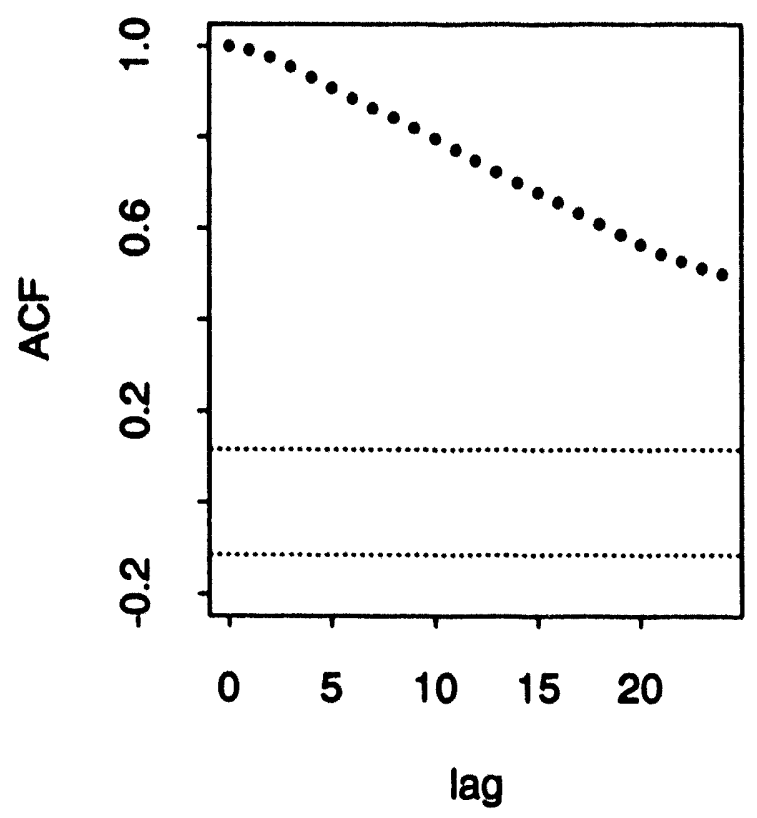

Fig. 1b. ACF of $X(t)$ versus lag.

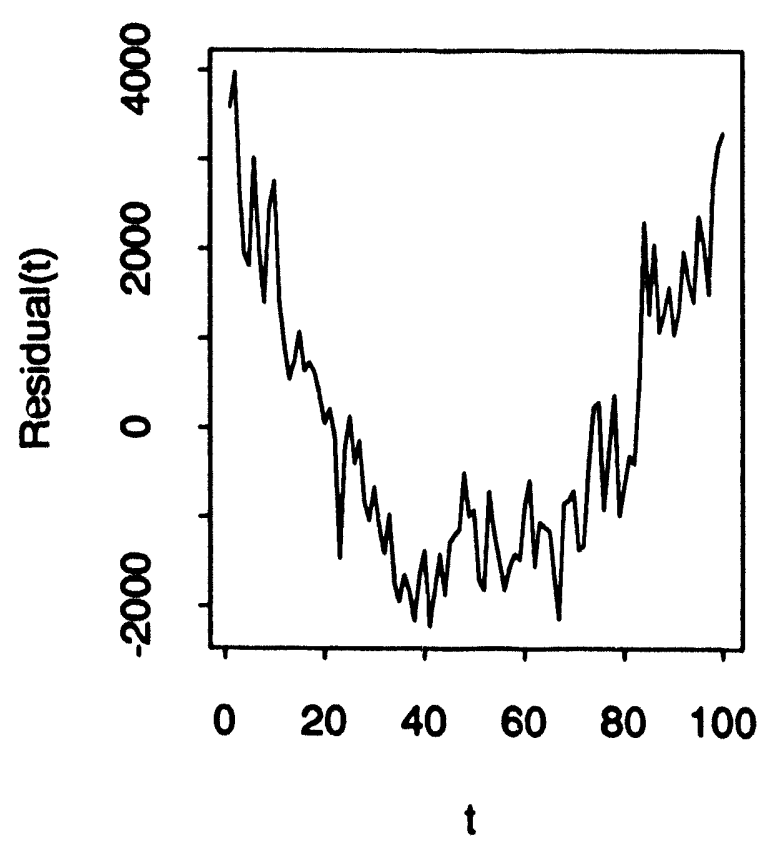

Fig. Id. Residuals from linear fit. 


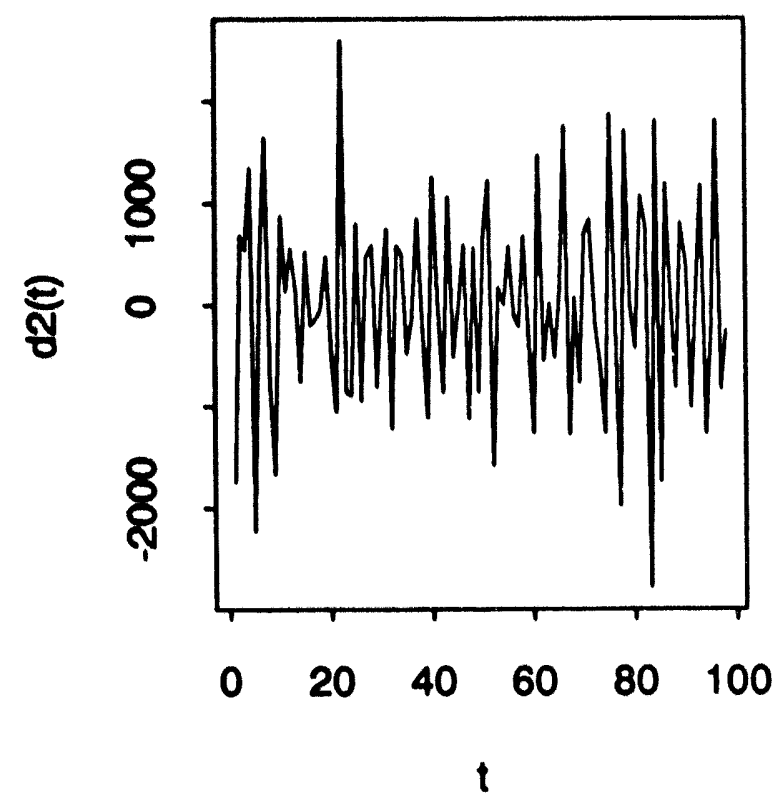

Fig. $2 a . d 2(t)=d 1(t)-d 1(t-1)$ versus $t$.

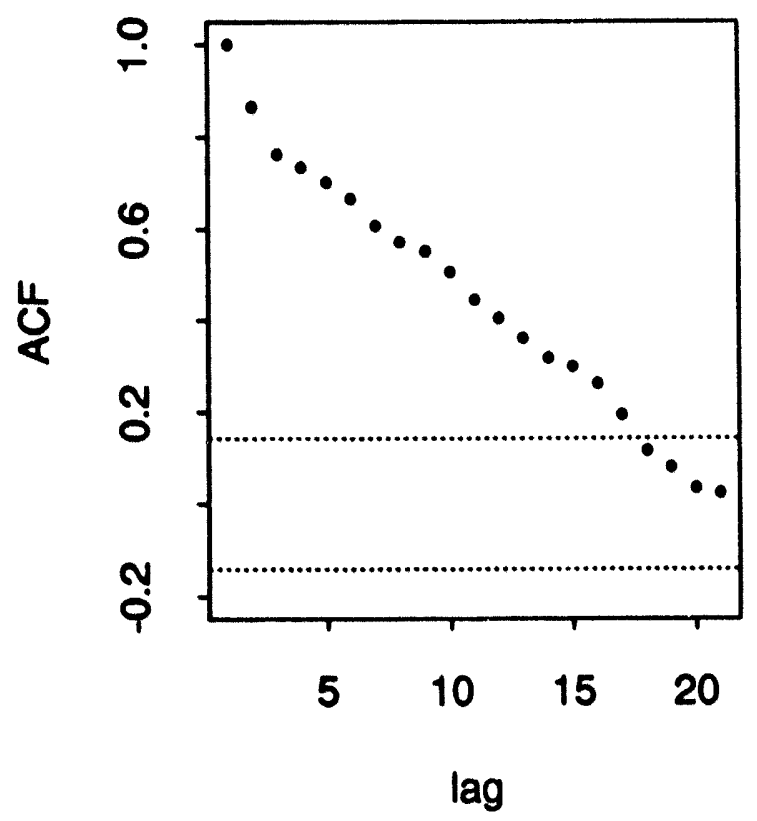

Fig. 2c. ACF of residuals from linear fit.

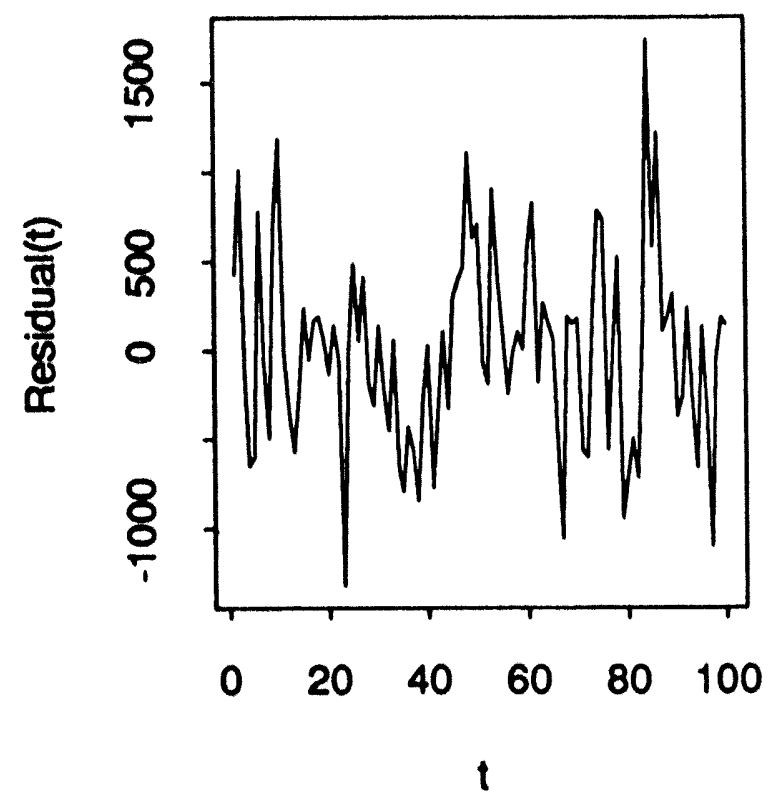

Fig. 2b. Residuals from quadratic fit.

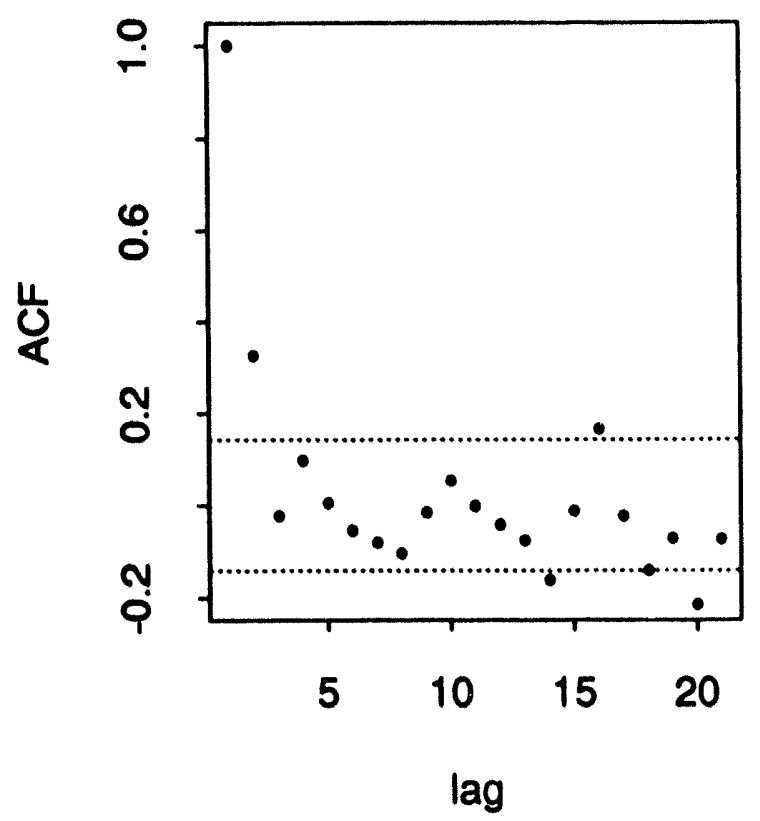

Fig. 2d. ACF of residuals from quadratic fit. 


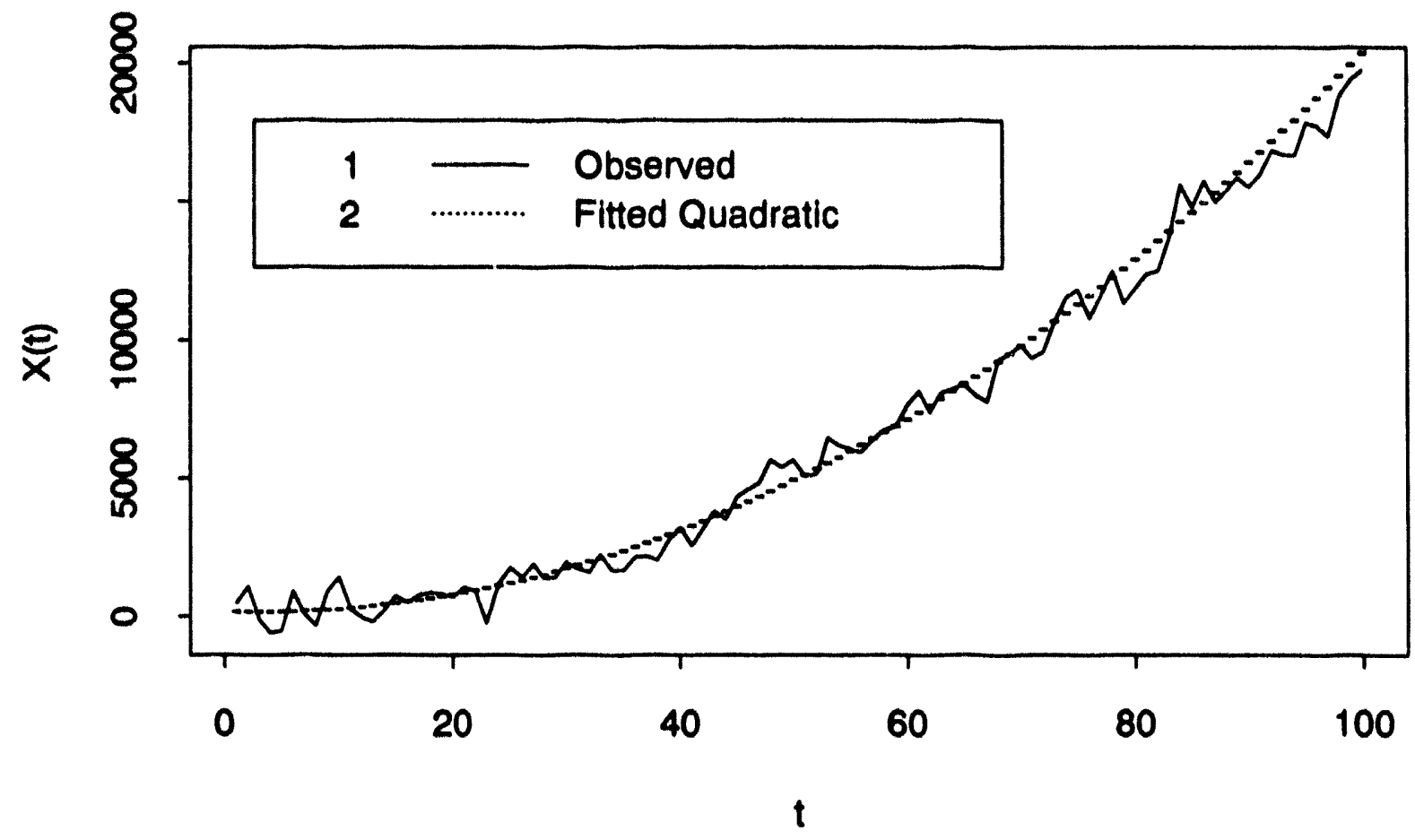

Fig. $3 a . X(t)$ versus $t$ with fit.

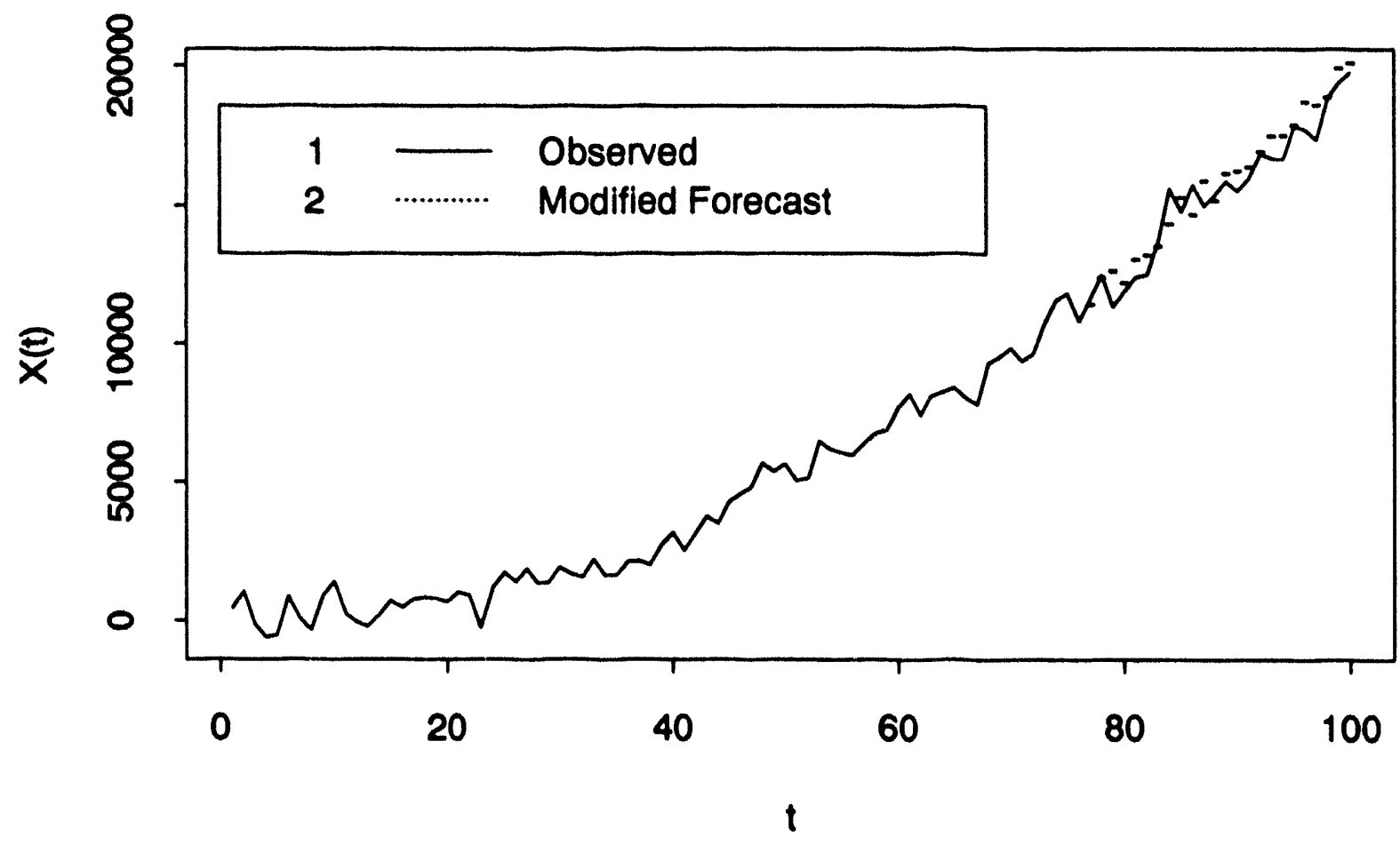

Fig. $3 b . X(t)$ versus $t$ with modified fit. 


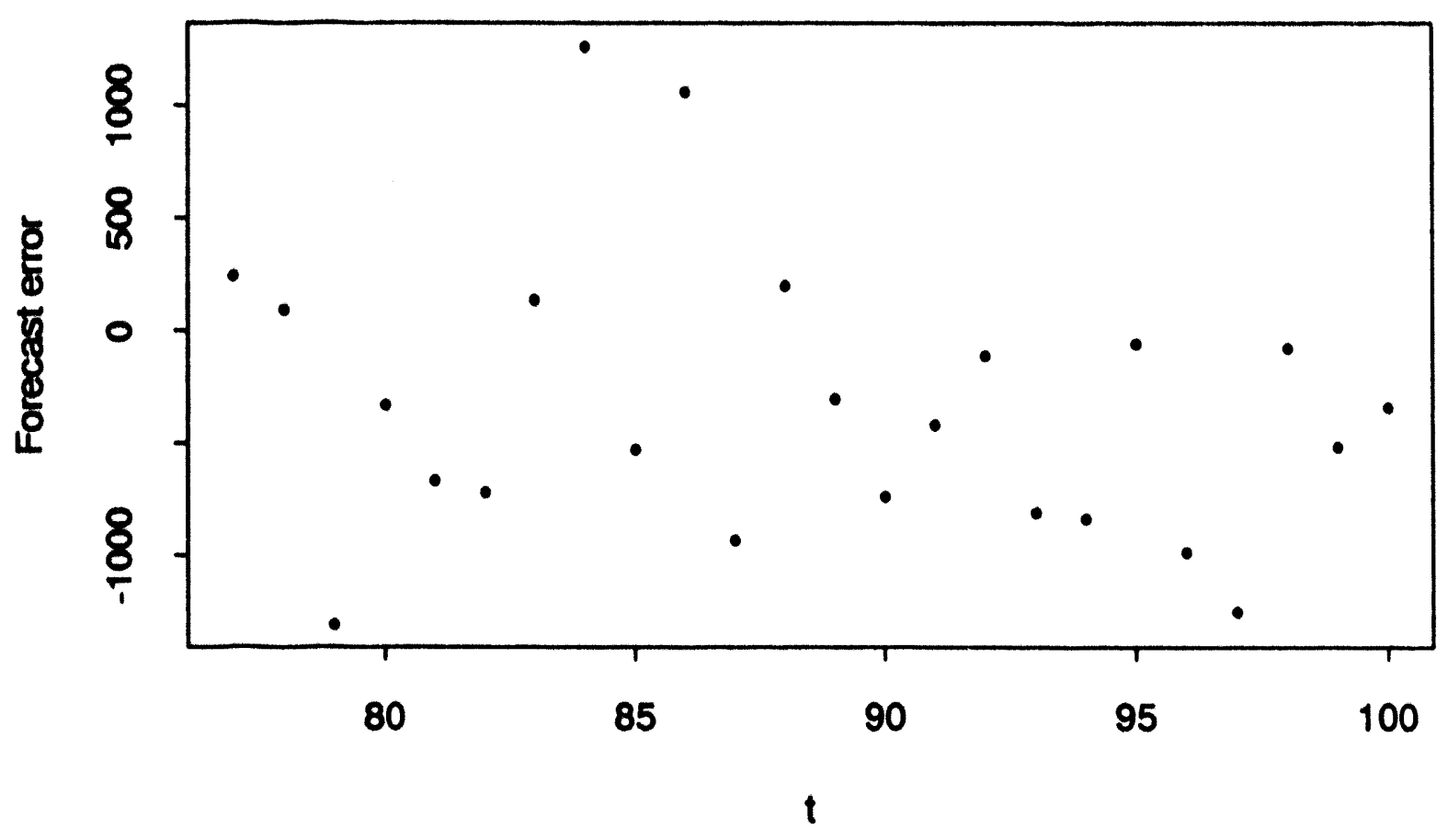

Fig. 4a. Forecast errors.

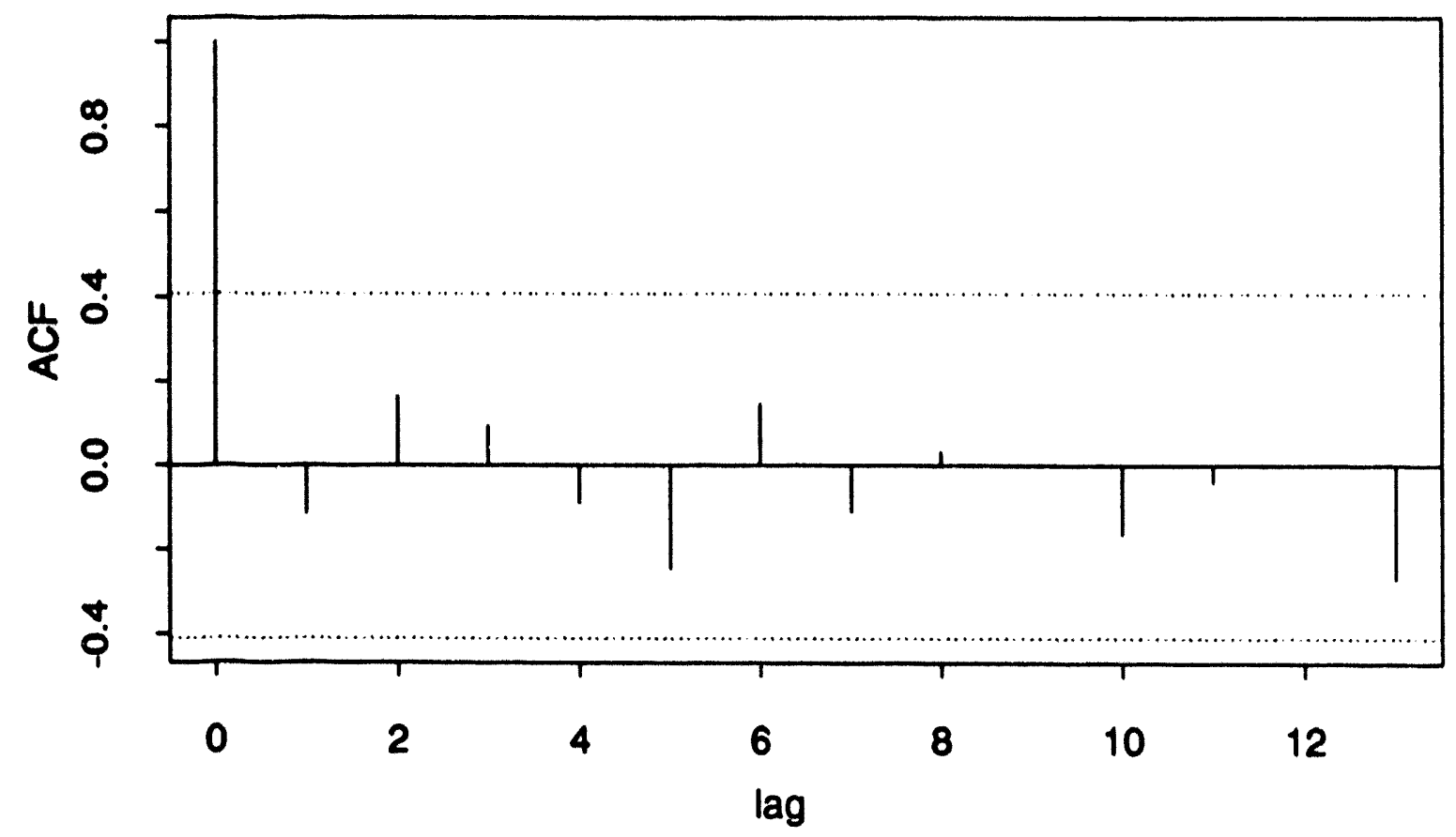

Fig. 4b. Sample ACF of forecast errors. 

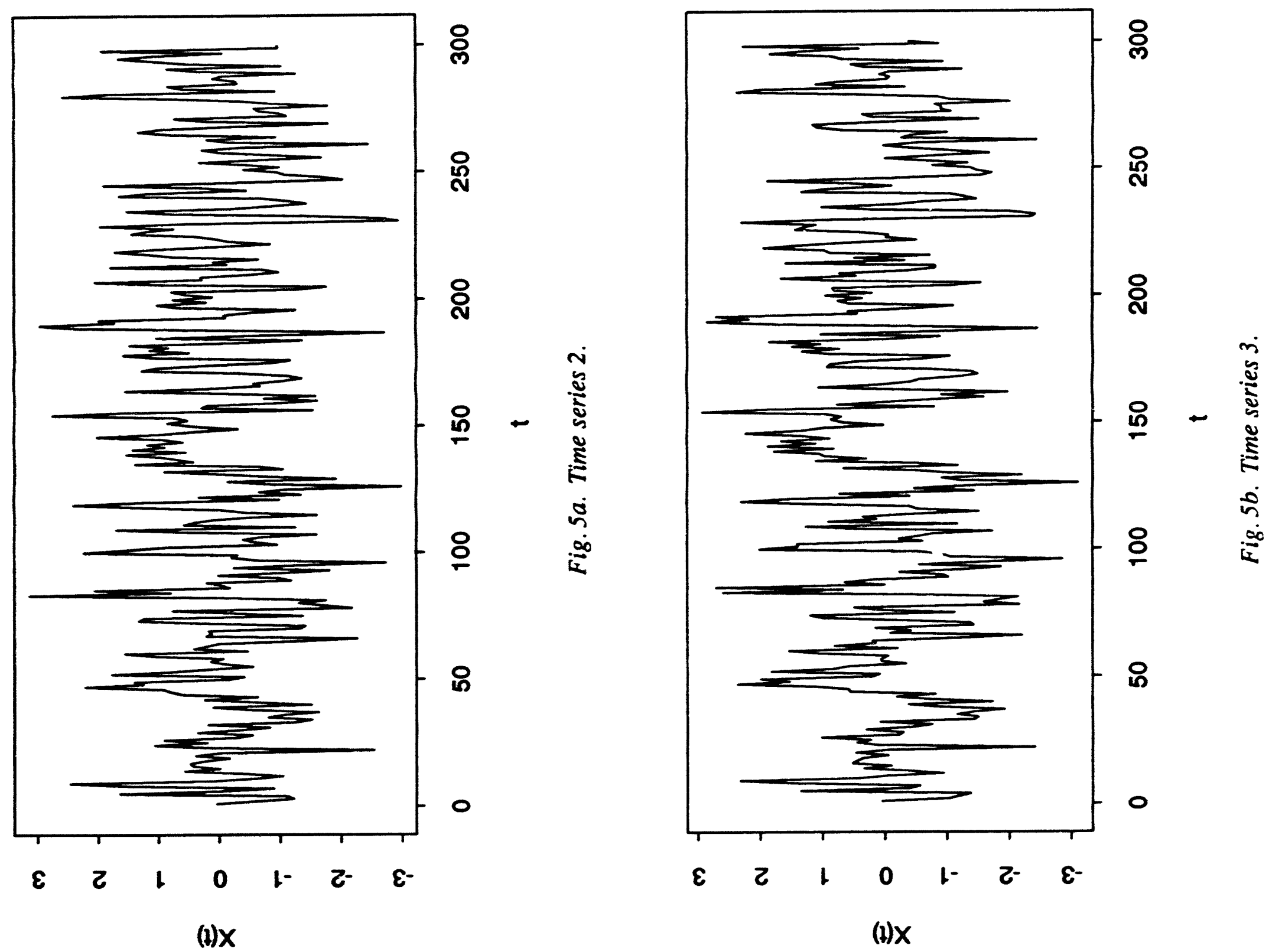

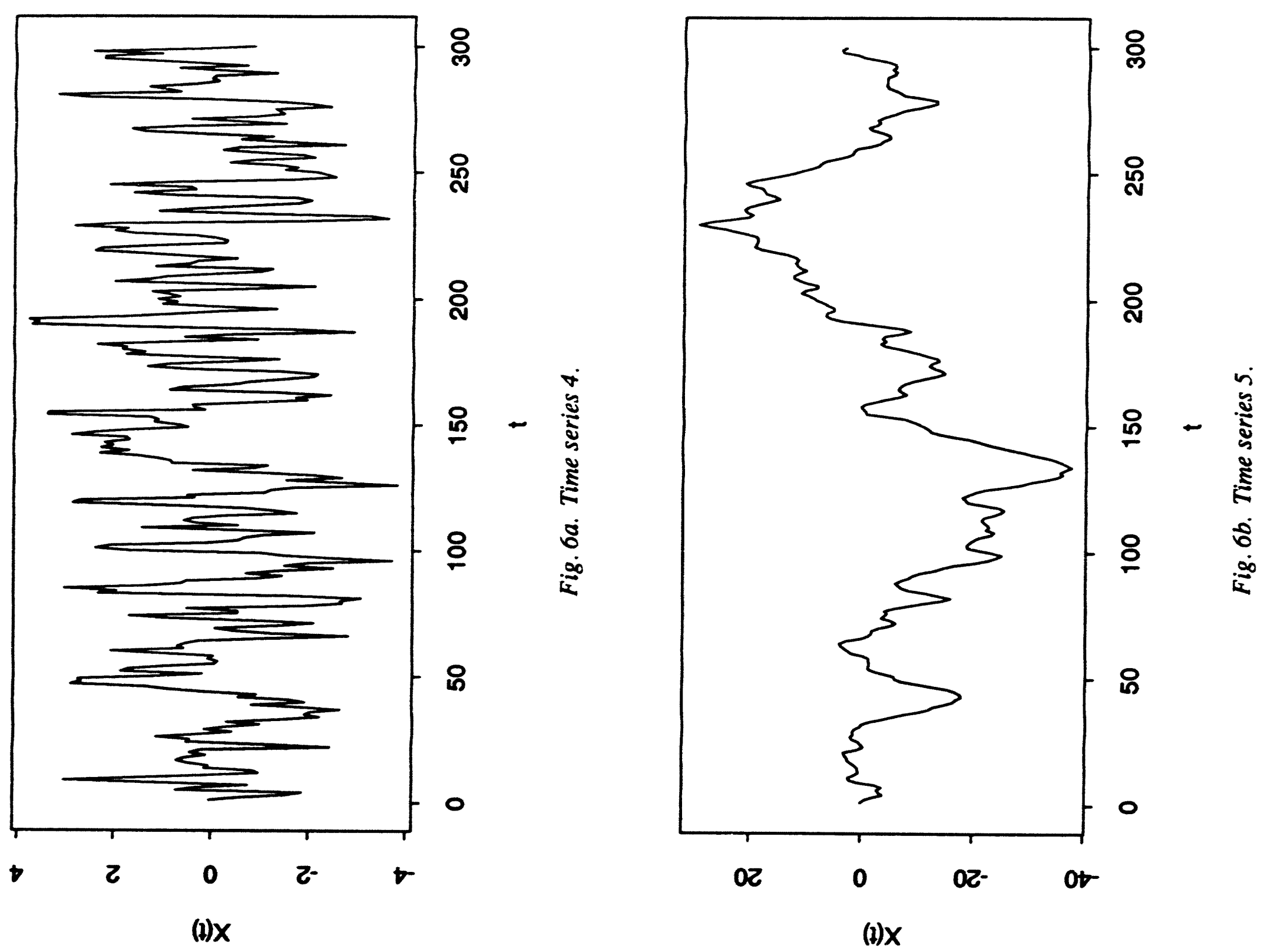


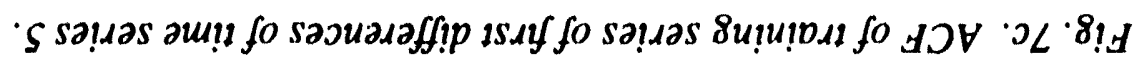

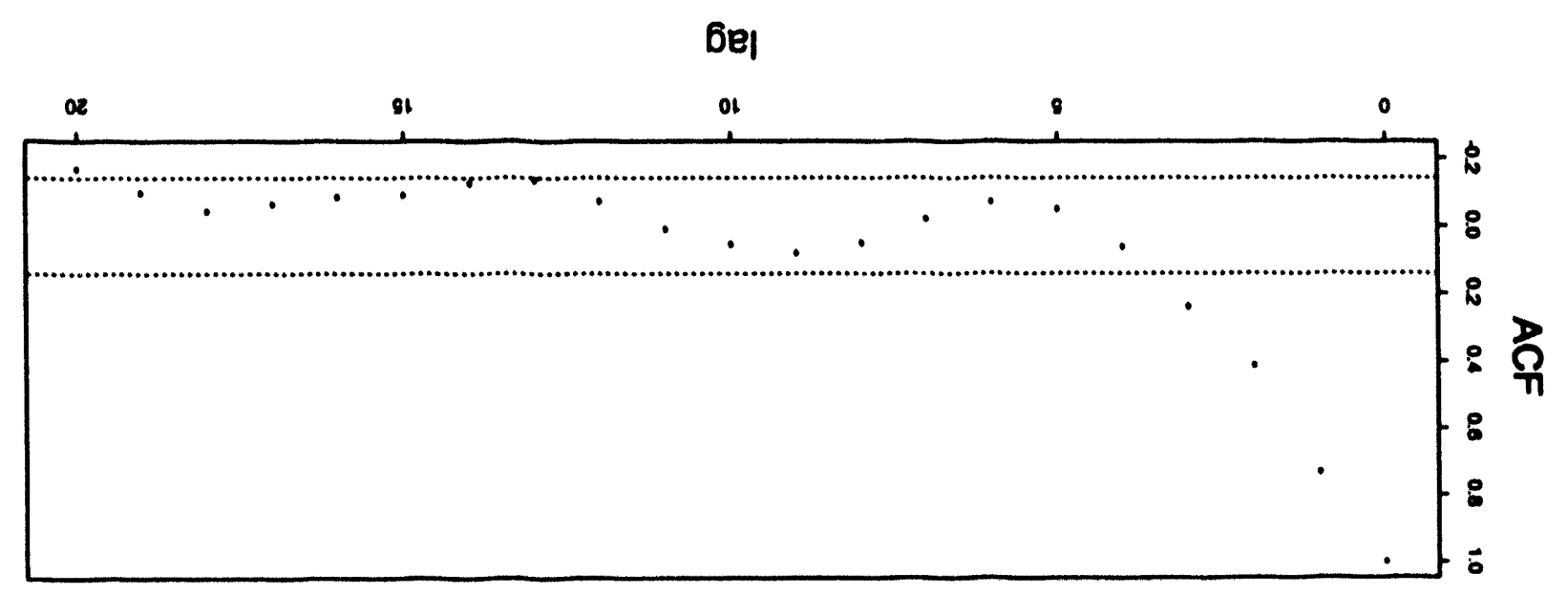

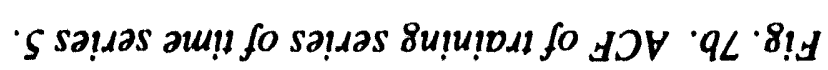

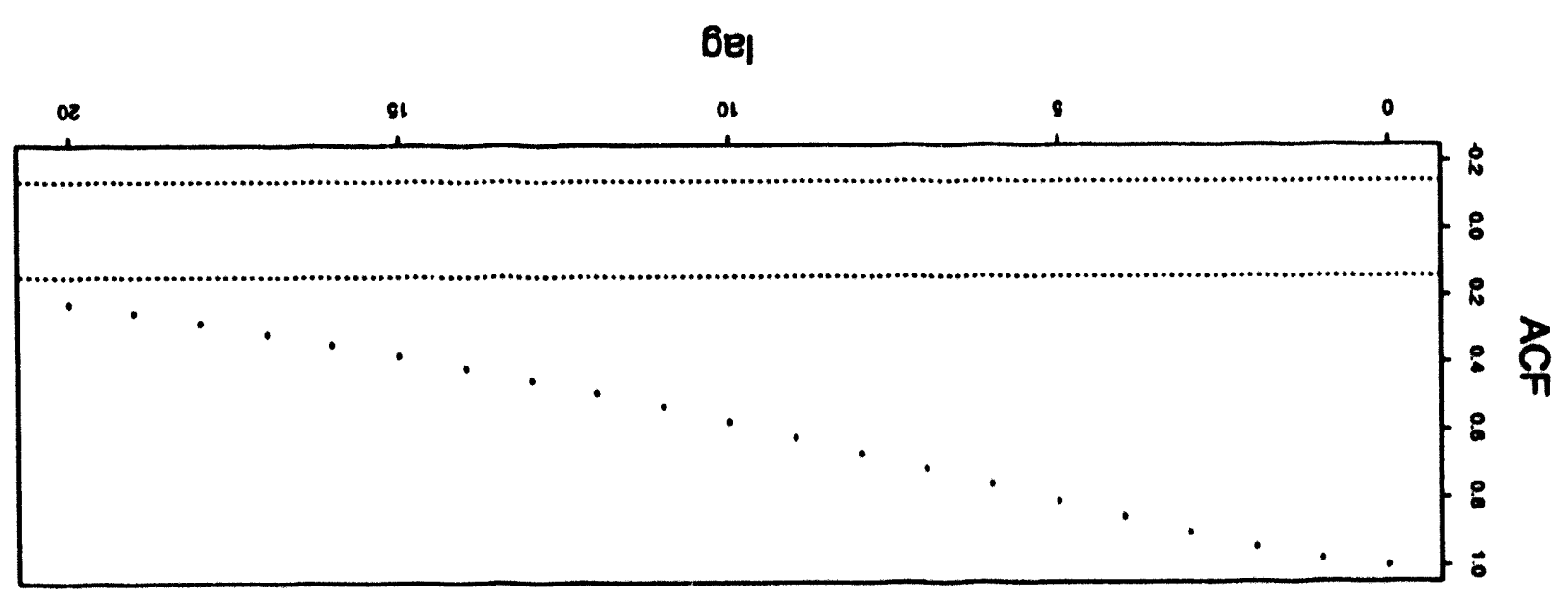

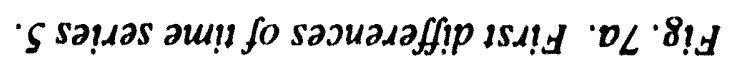

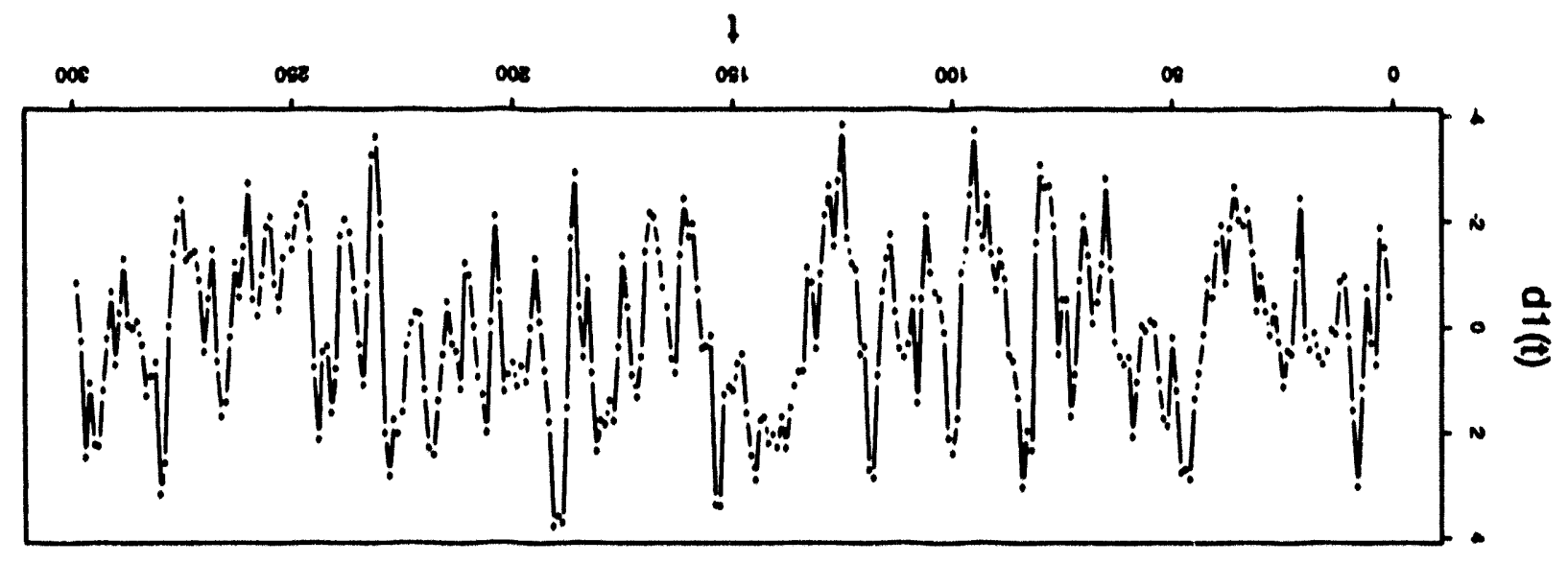




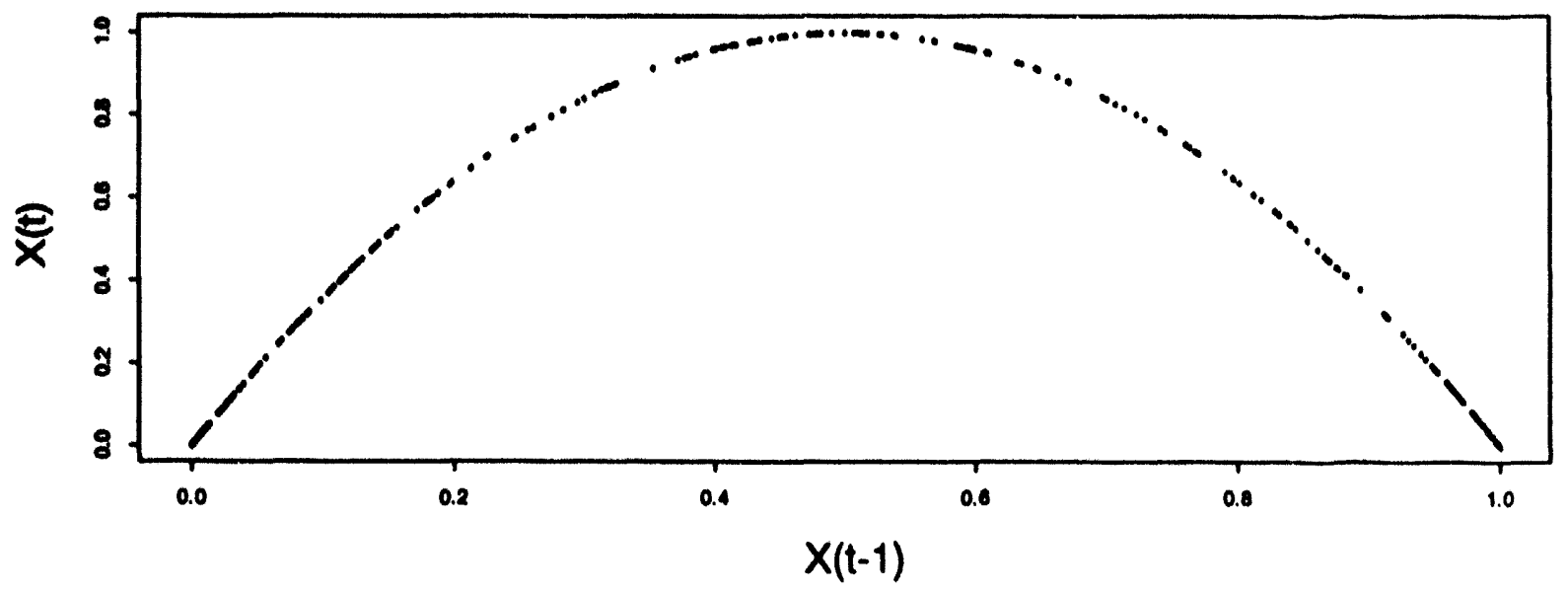

Fig. 8a. Error variance $=0$.

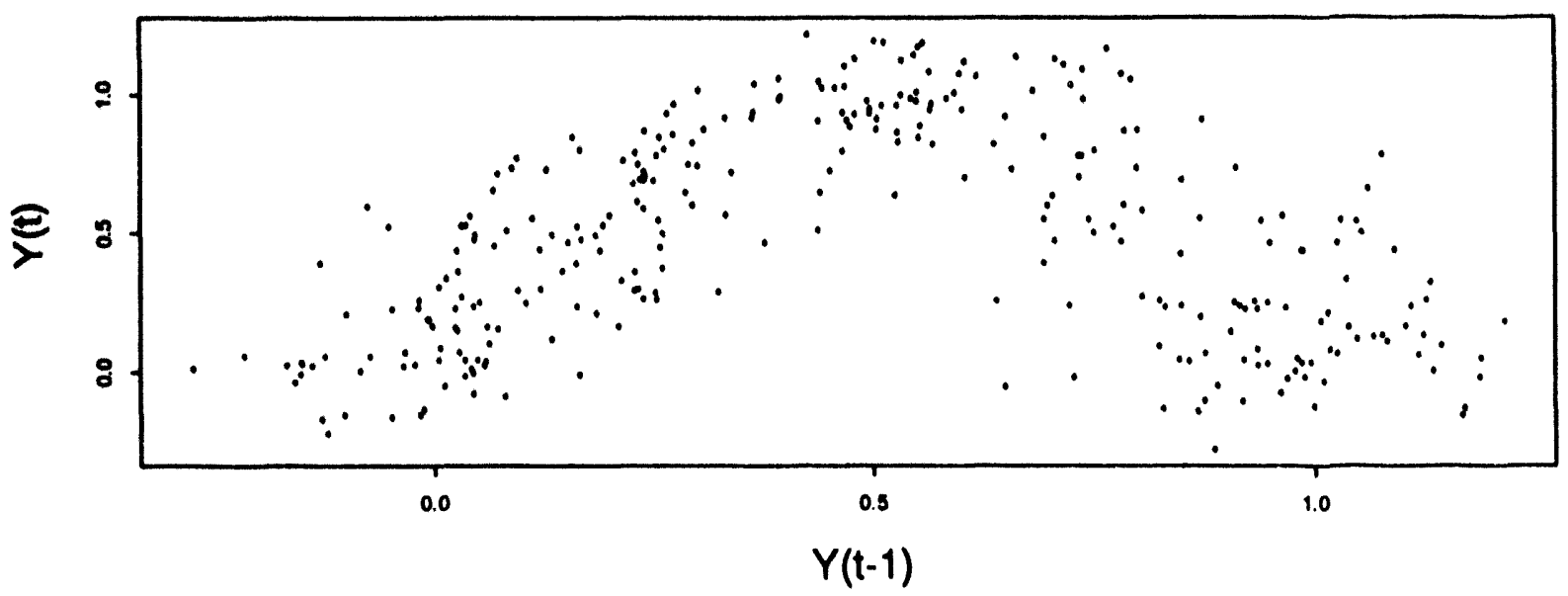

Fig. 8b. Error variance $=0.01$.

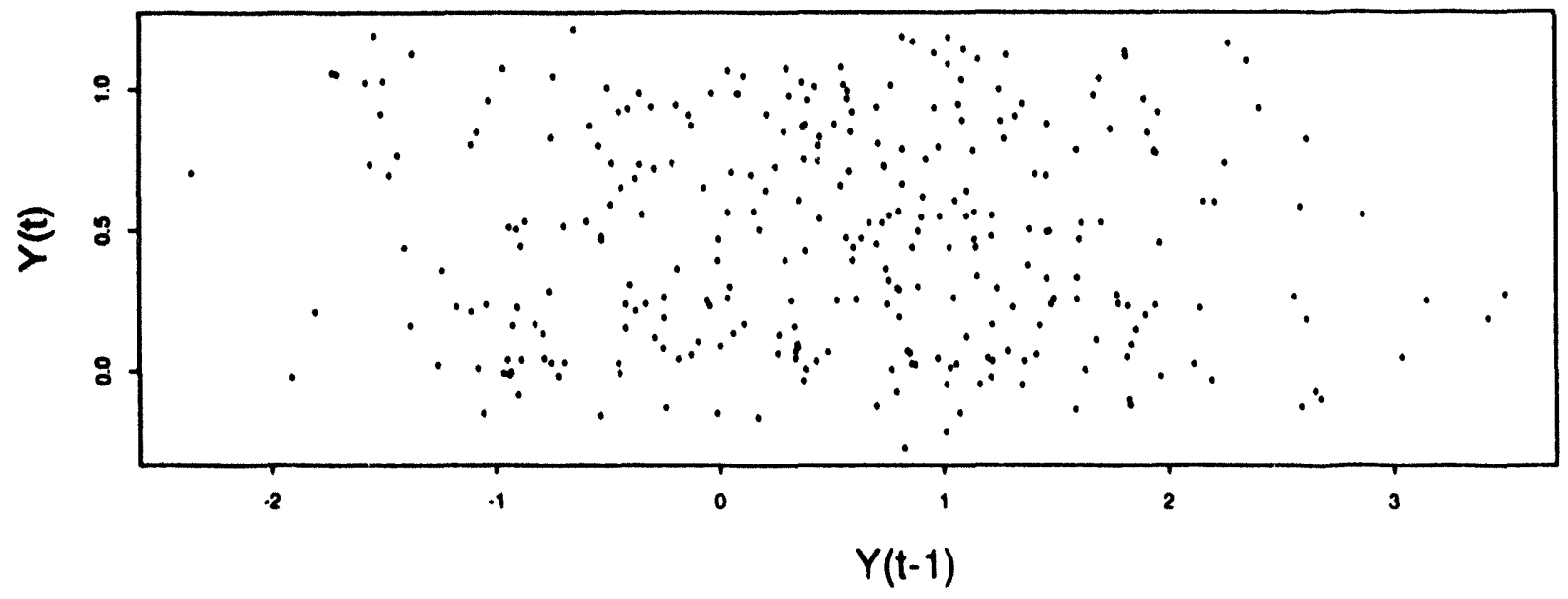

Fig. 8c. Error variance $=1$. 


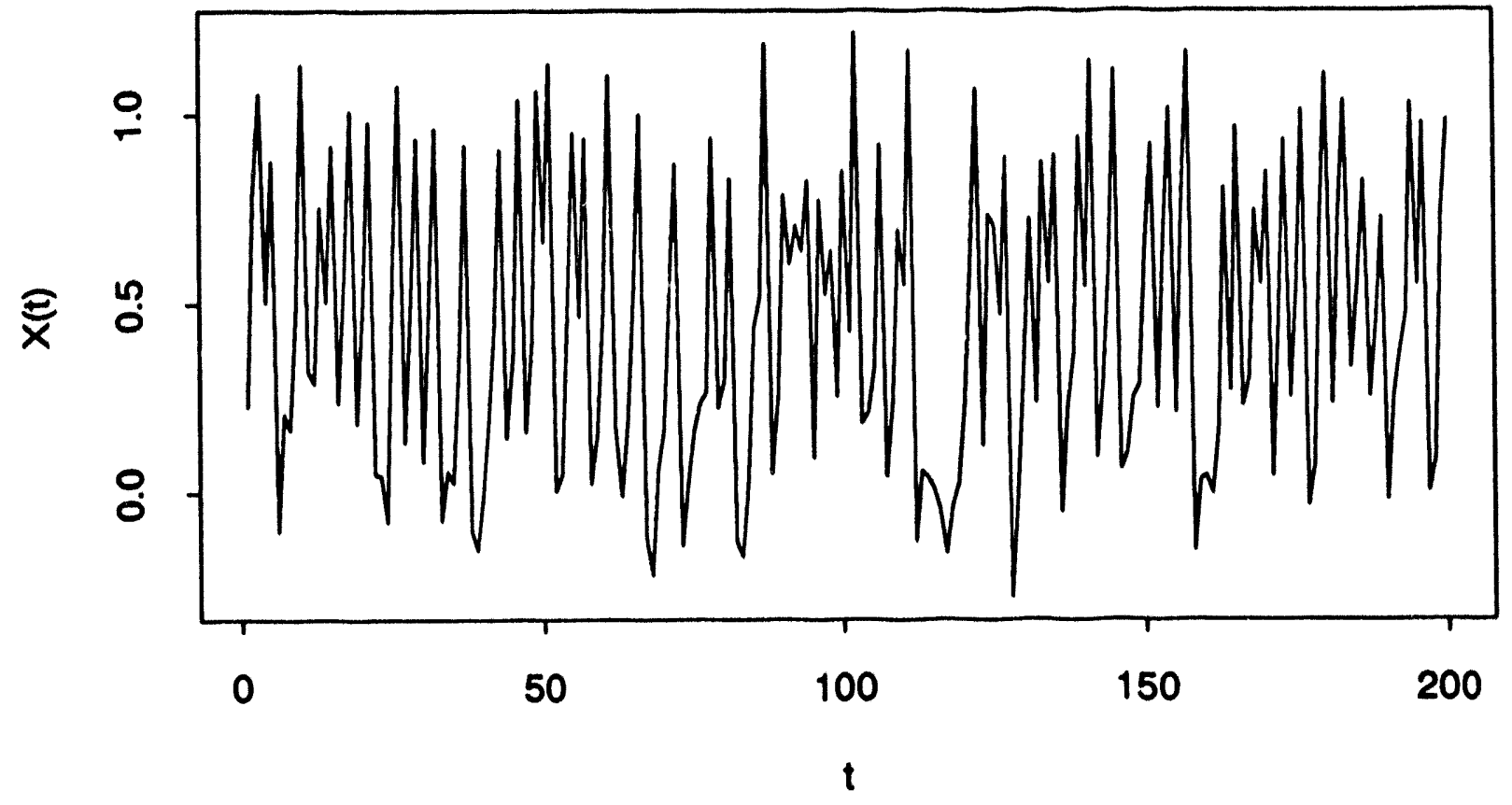

Fig. 9a. Time series plot of series 6 .

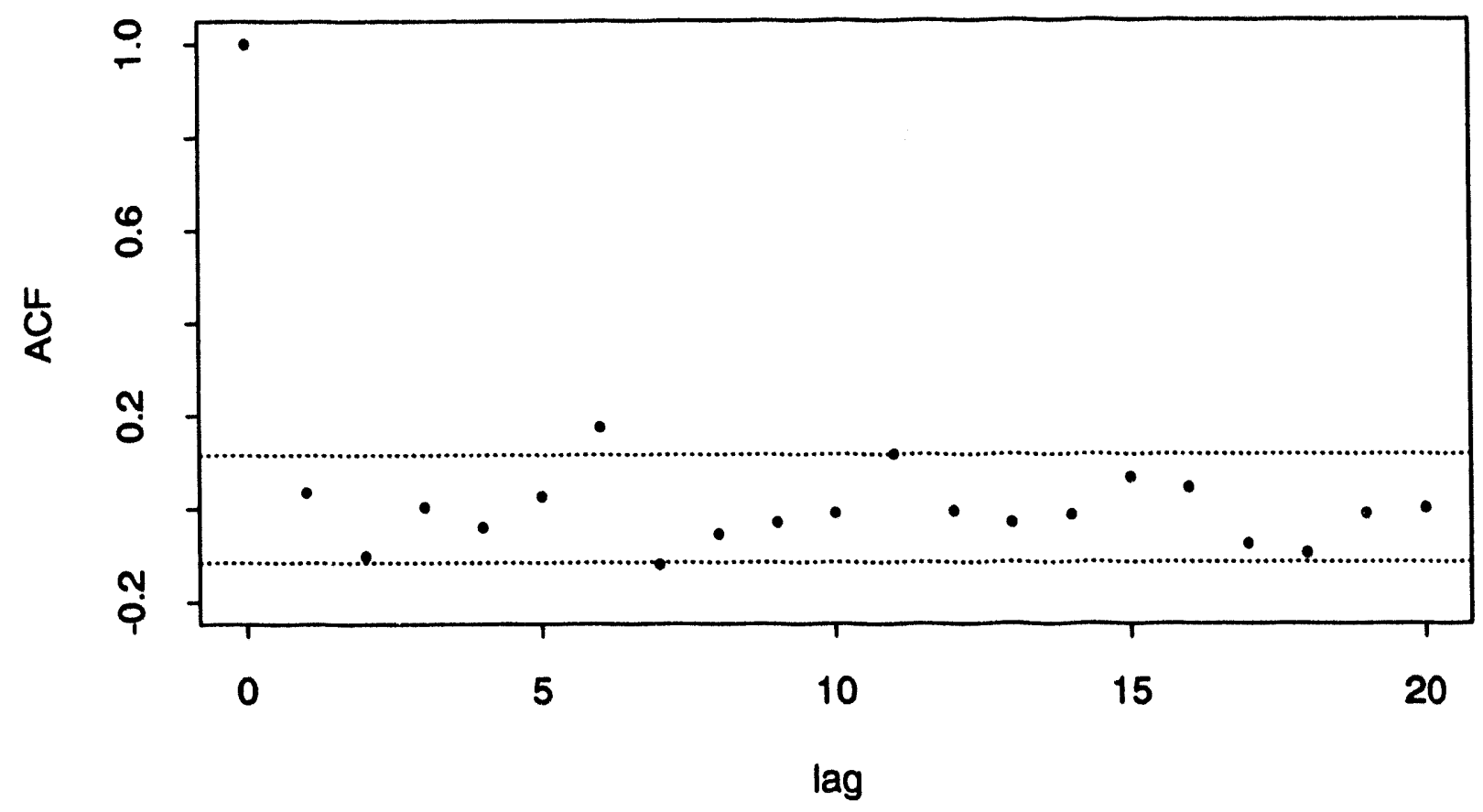

Fig. 9b. ACF of $X(t)$ versus lag. 


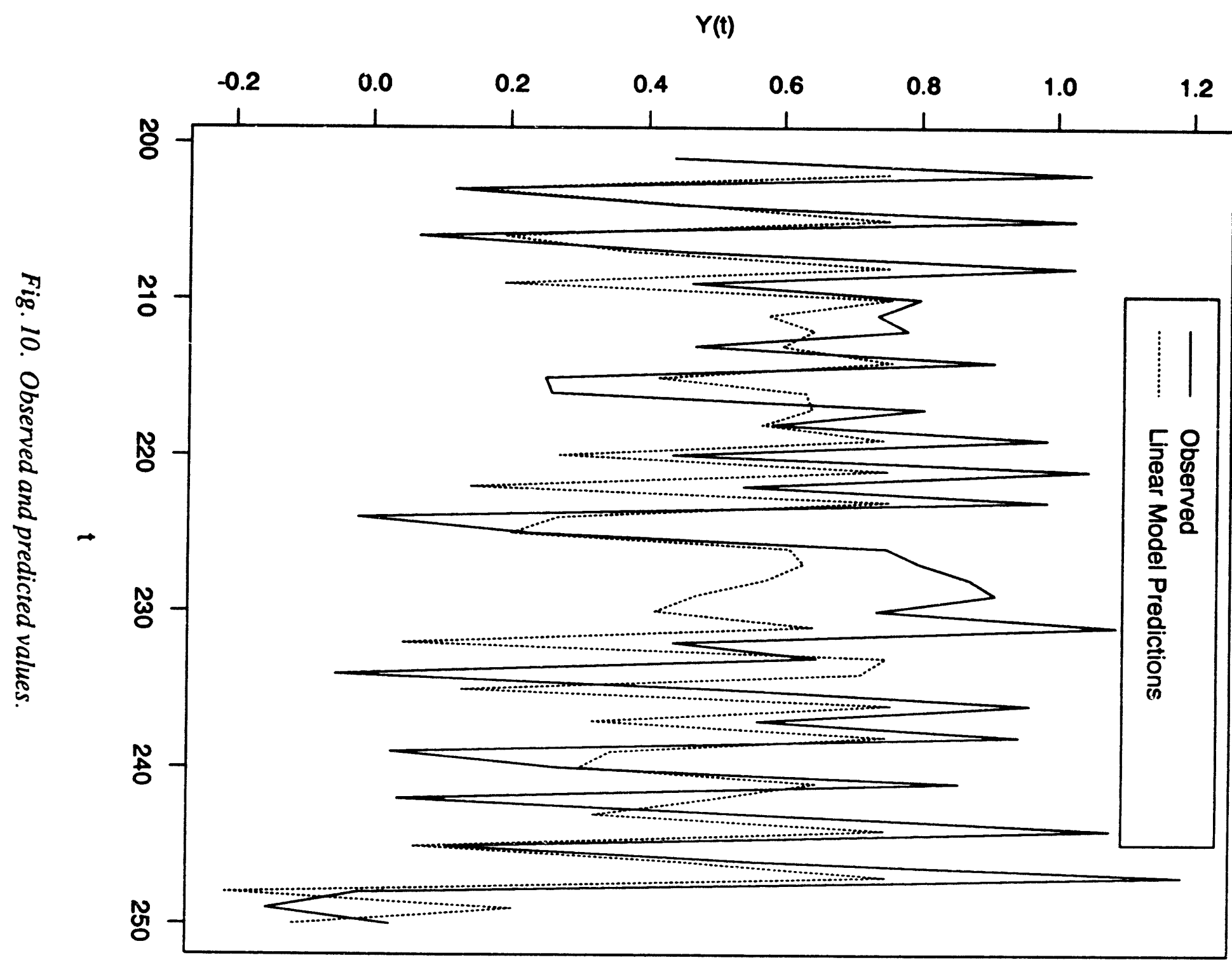




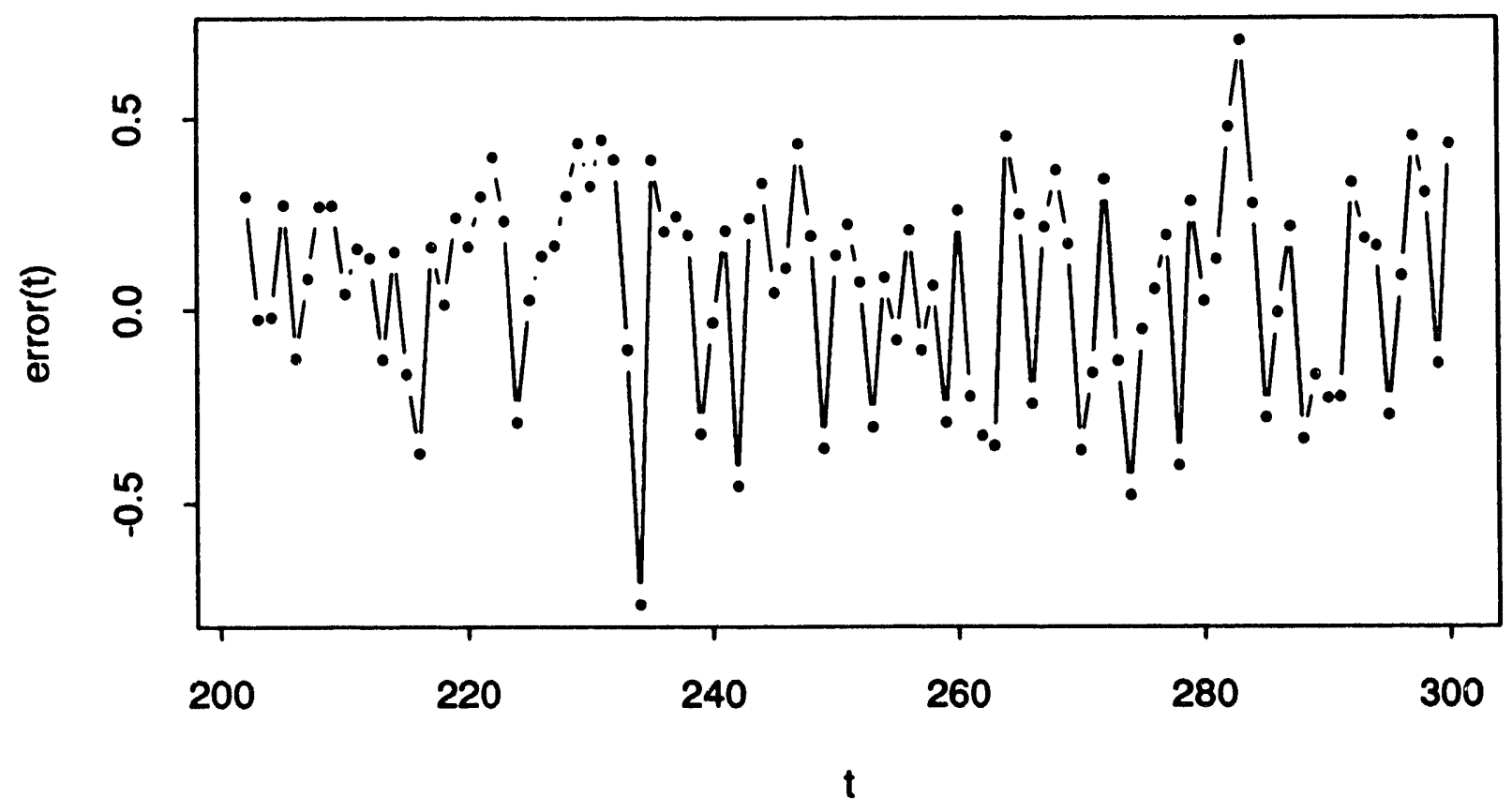

Fig. 11a. Linear model forecast error.

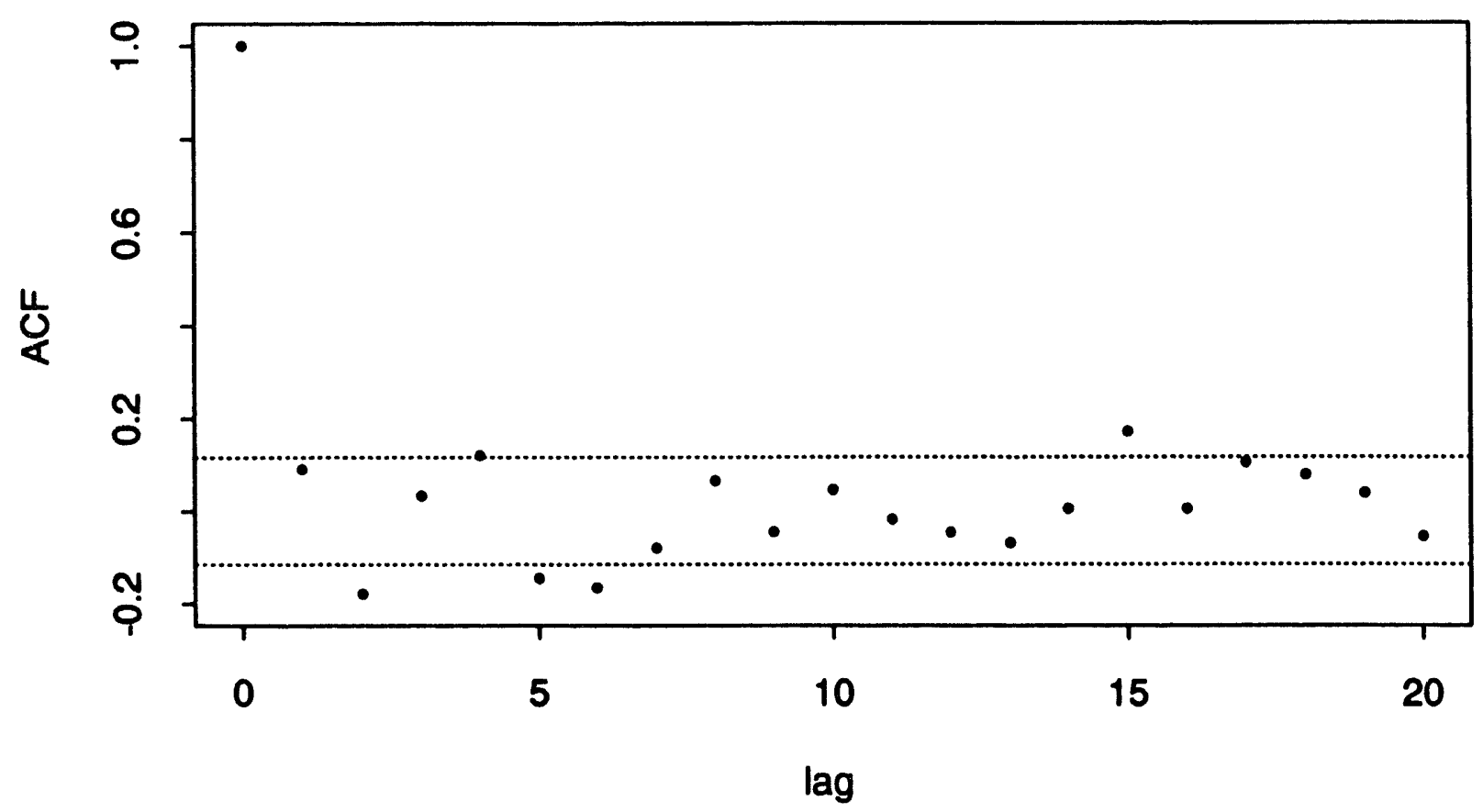

Fig. 11b. ACF versus lag of linear model forecast errors. 


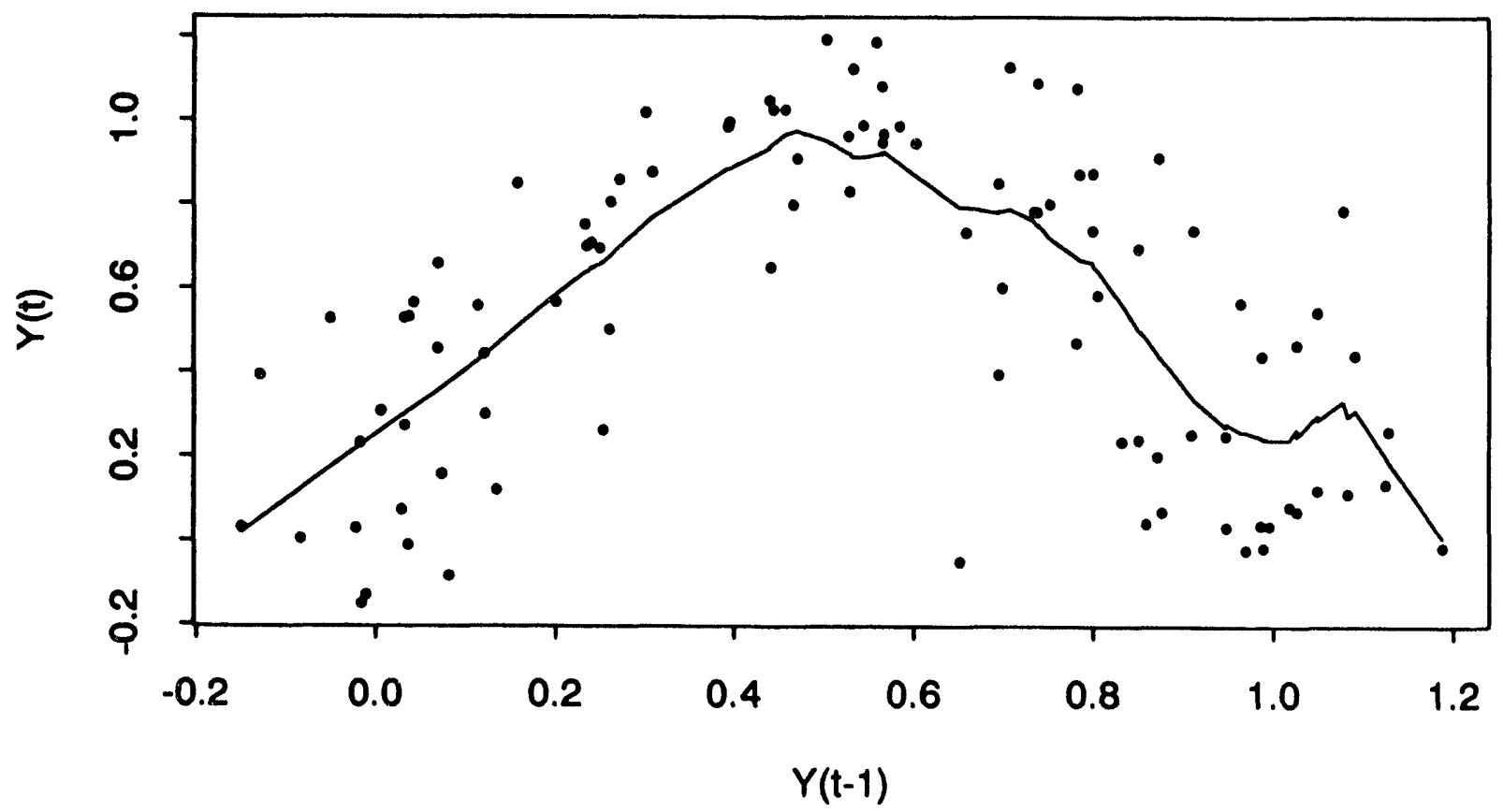

Fig. 12a. Solid line is the nonlinear fit.

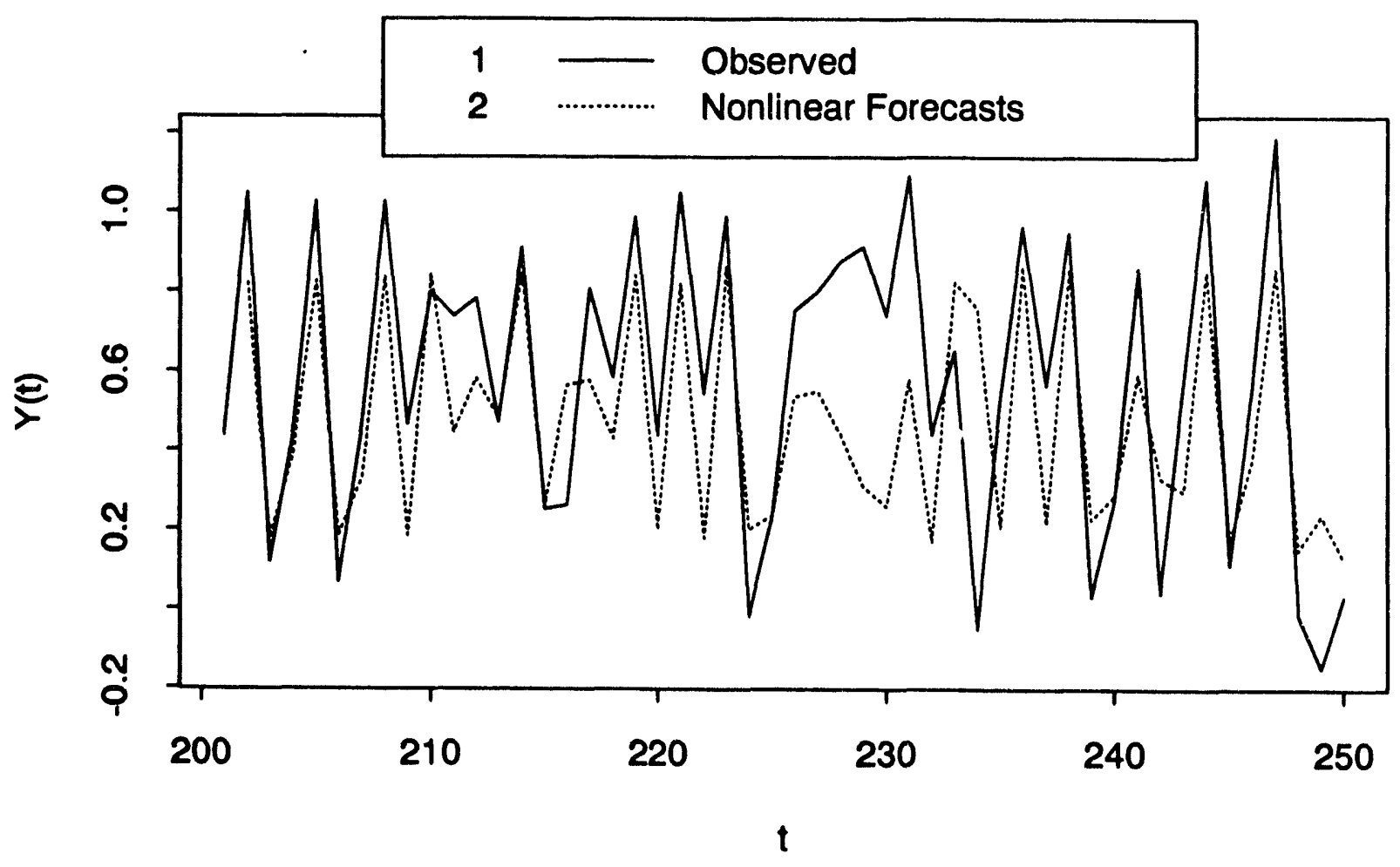

Fig. 12b. Observed and nonlinear model forecasts. 

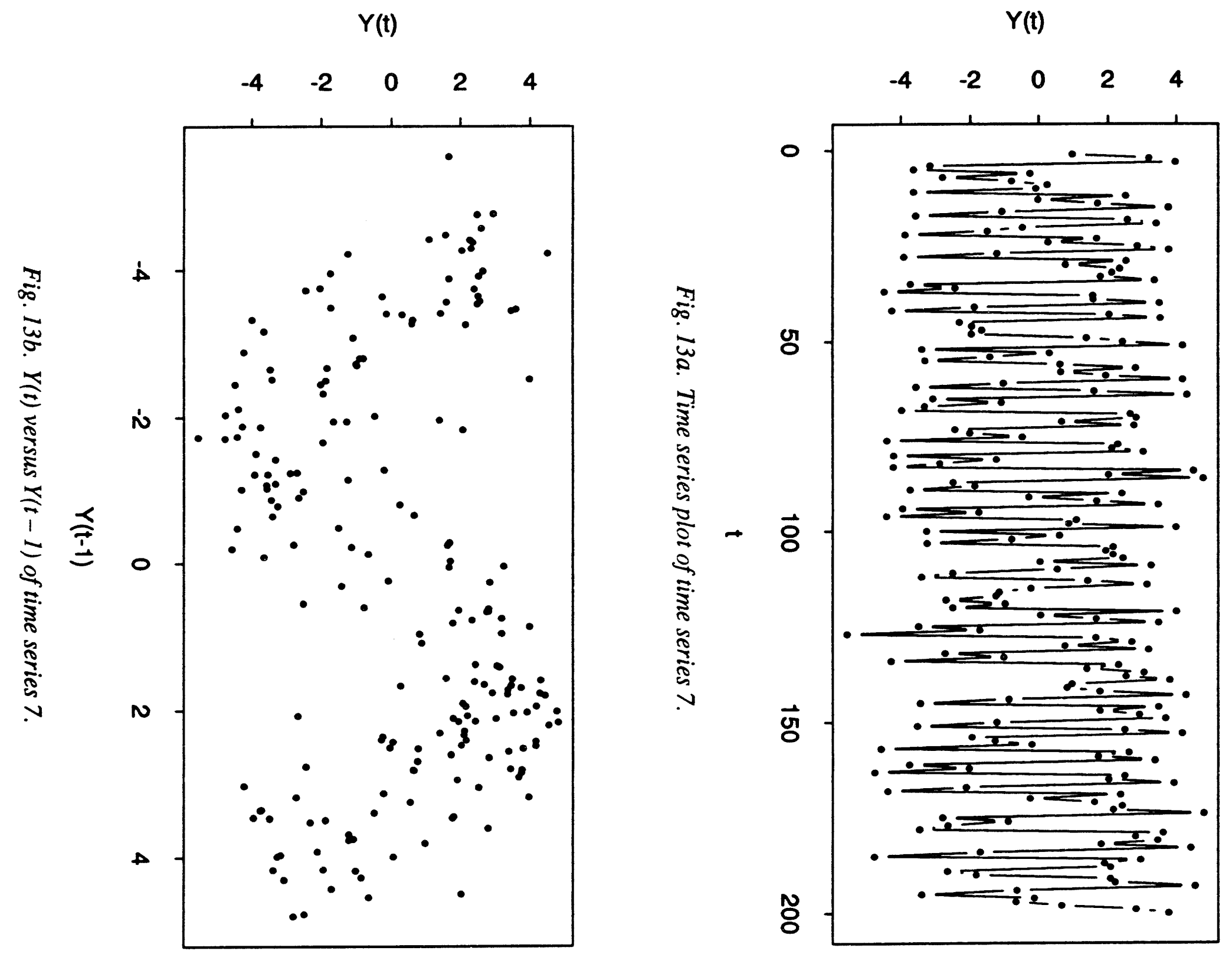


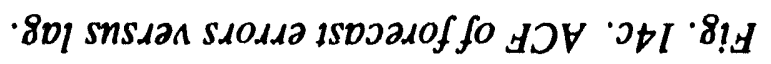

6e|

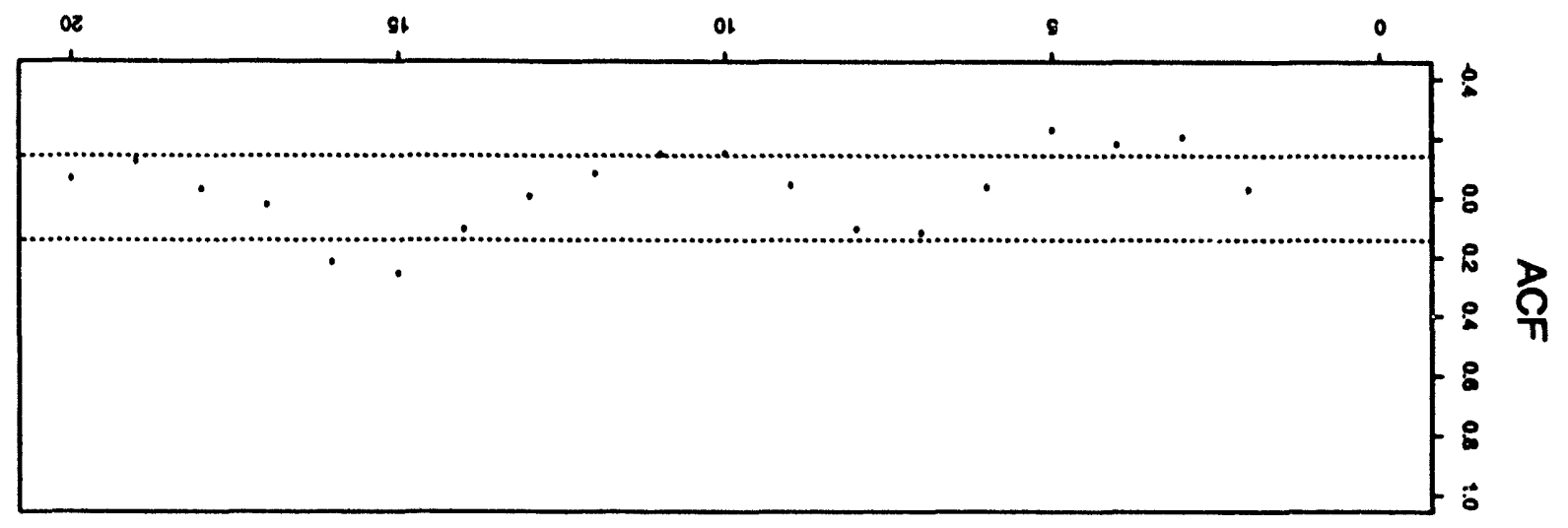

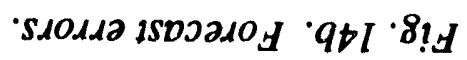

1

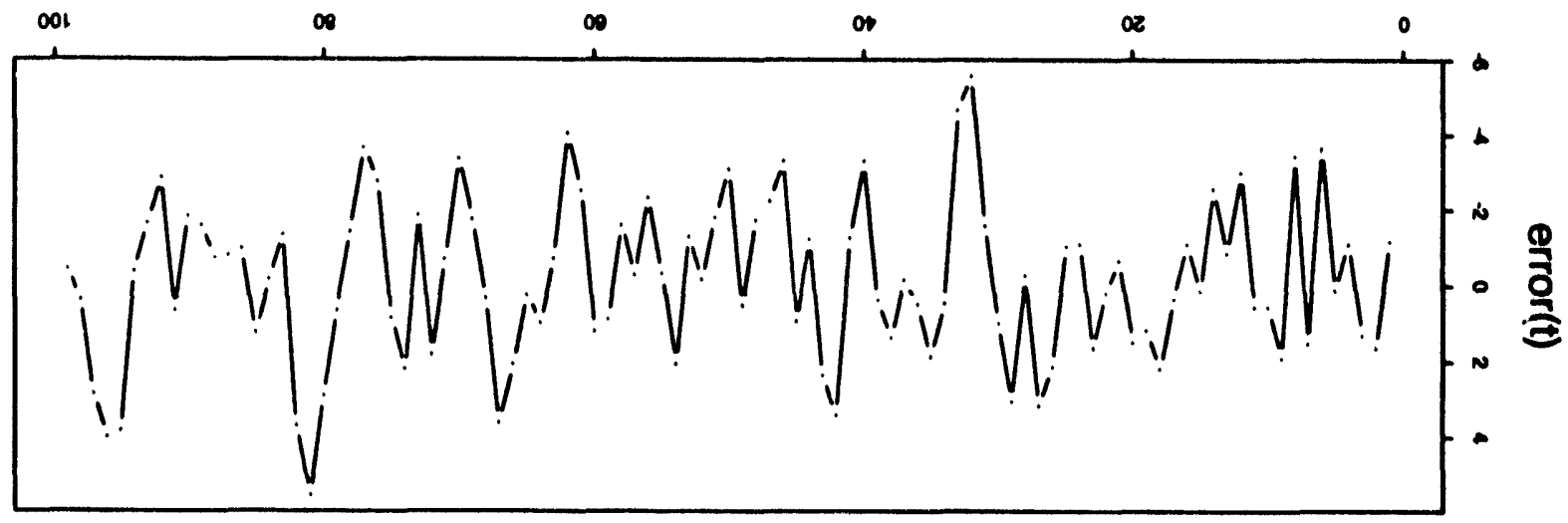

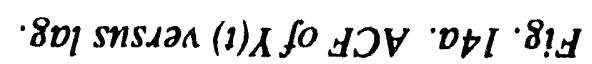

$6 \mathrm{e}$

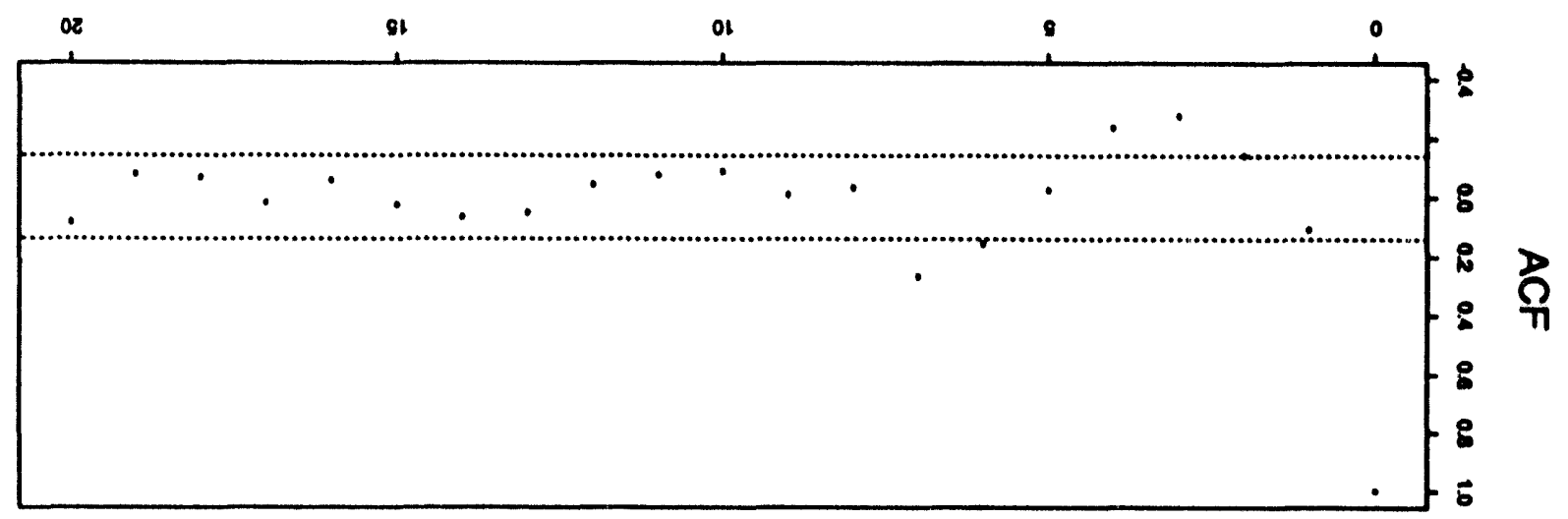




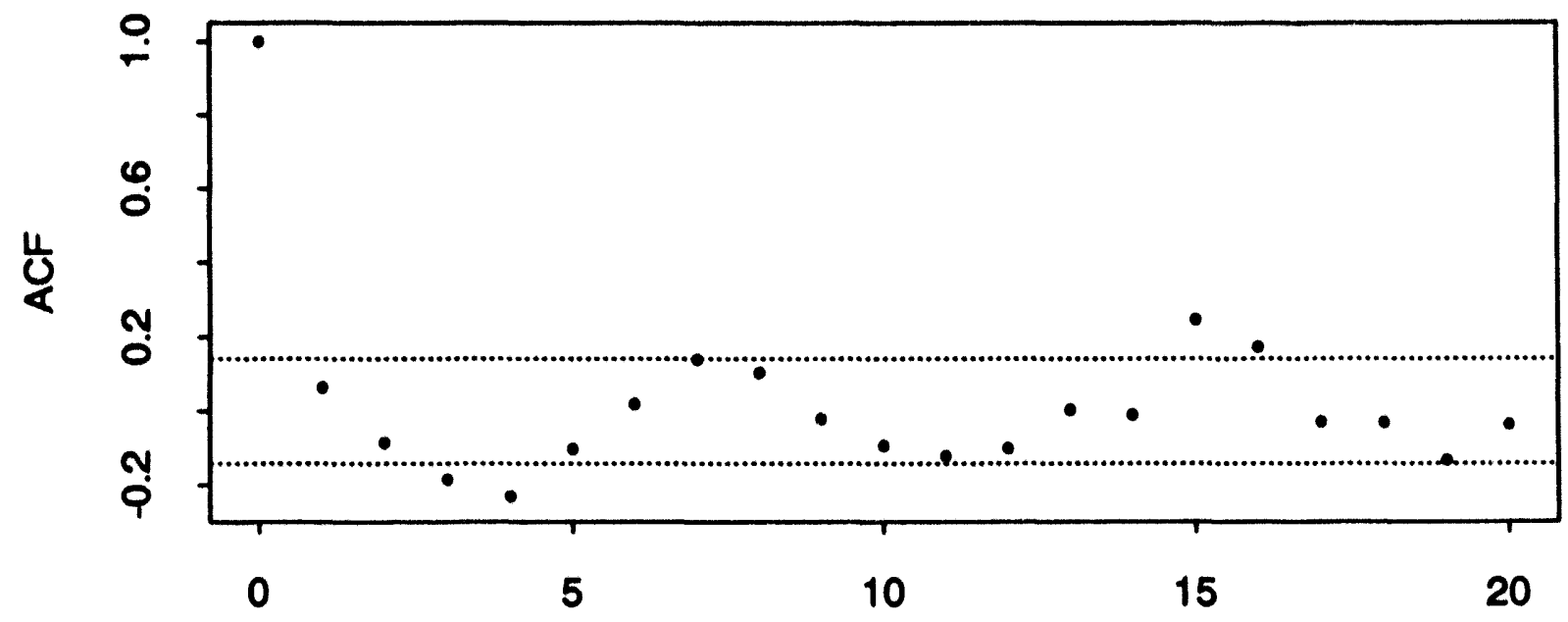

lag

Fig. 15a. ACF of lag-1 nonlinear model errors versus lag.

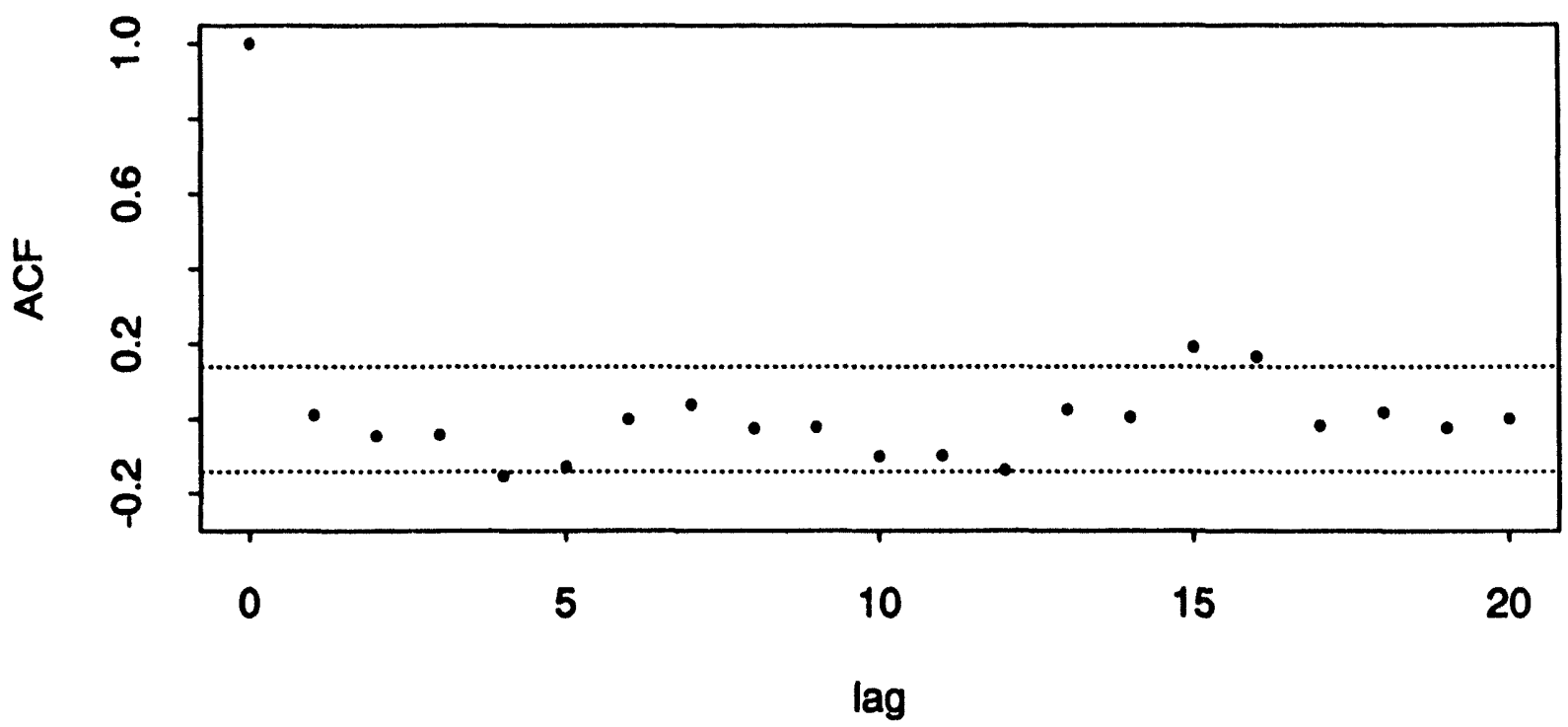

Fig. 15b. ACF of lag-2 nonlinear model errors versus lag. 


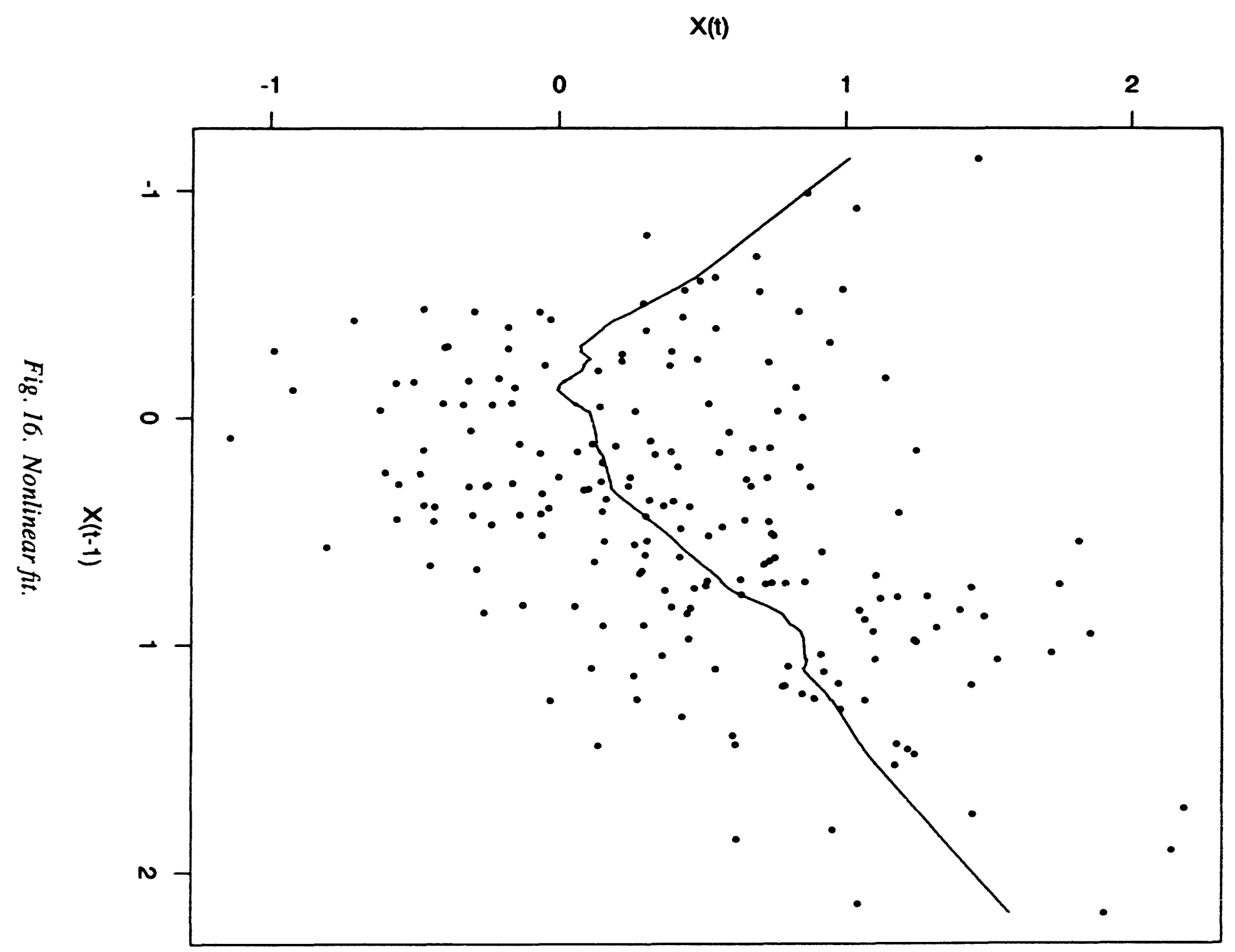




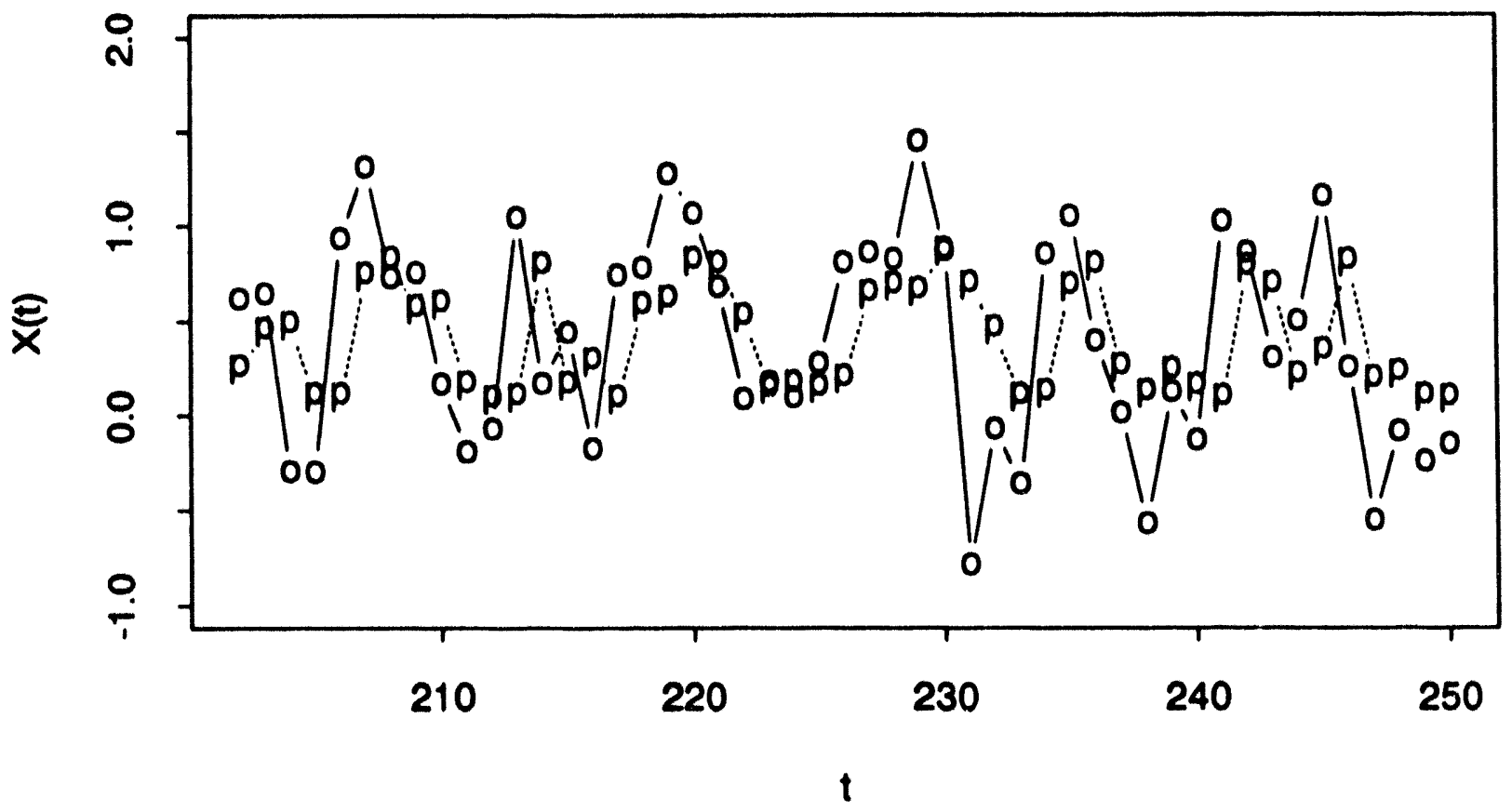

Fig. 17a. o-observed, $p$ - predicted.

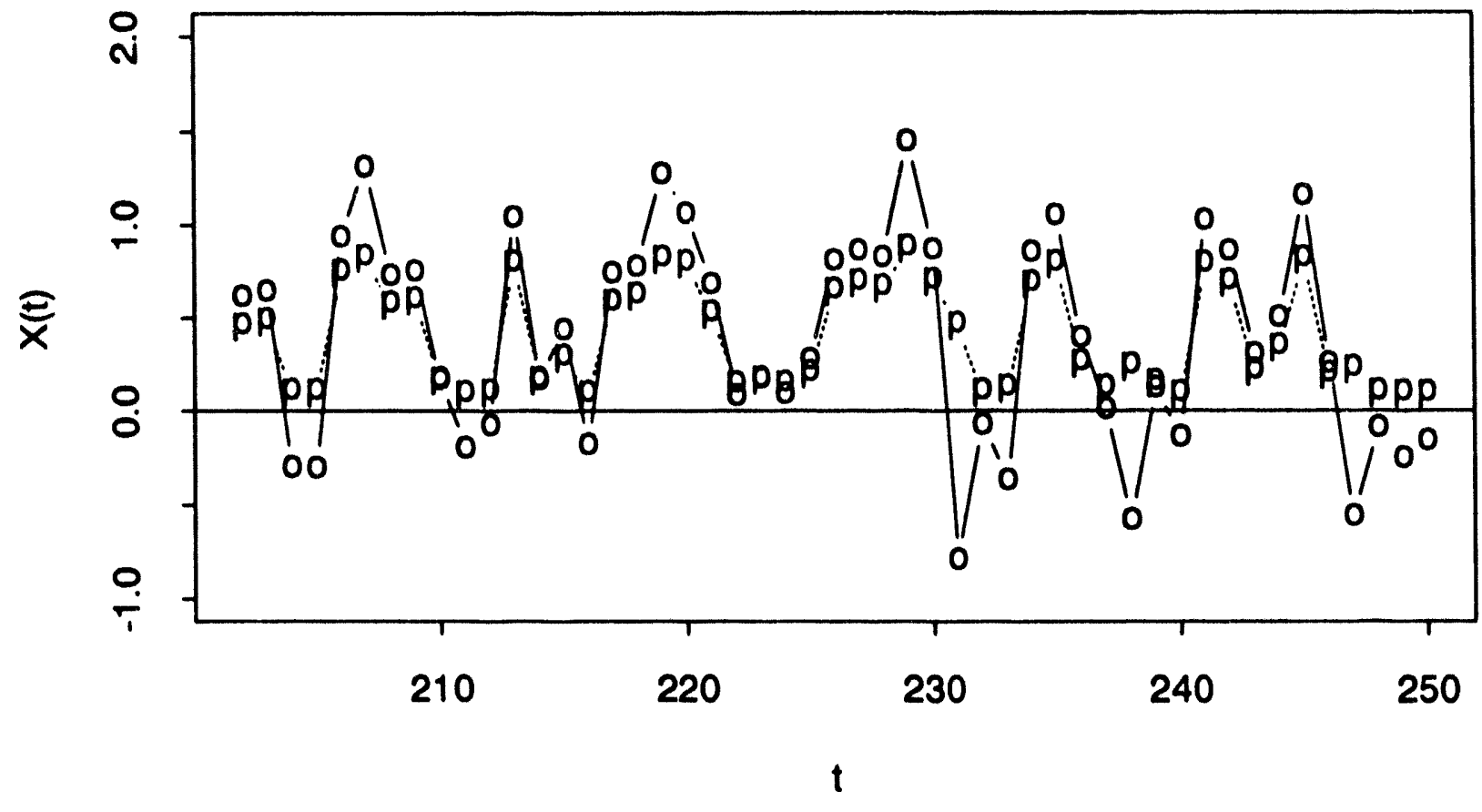

Fig. 17b. o-observed, $p$ - prediction of next observation. 


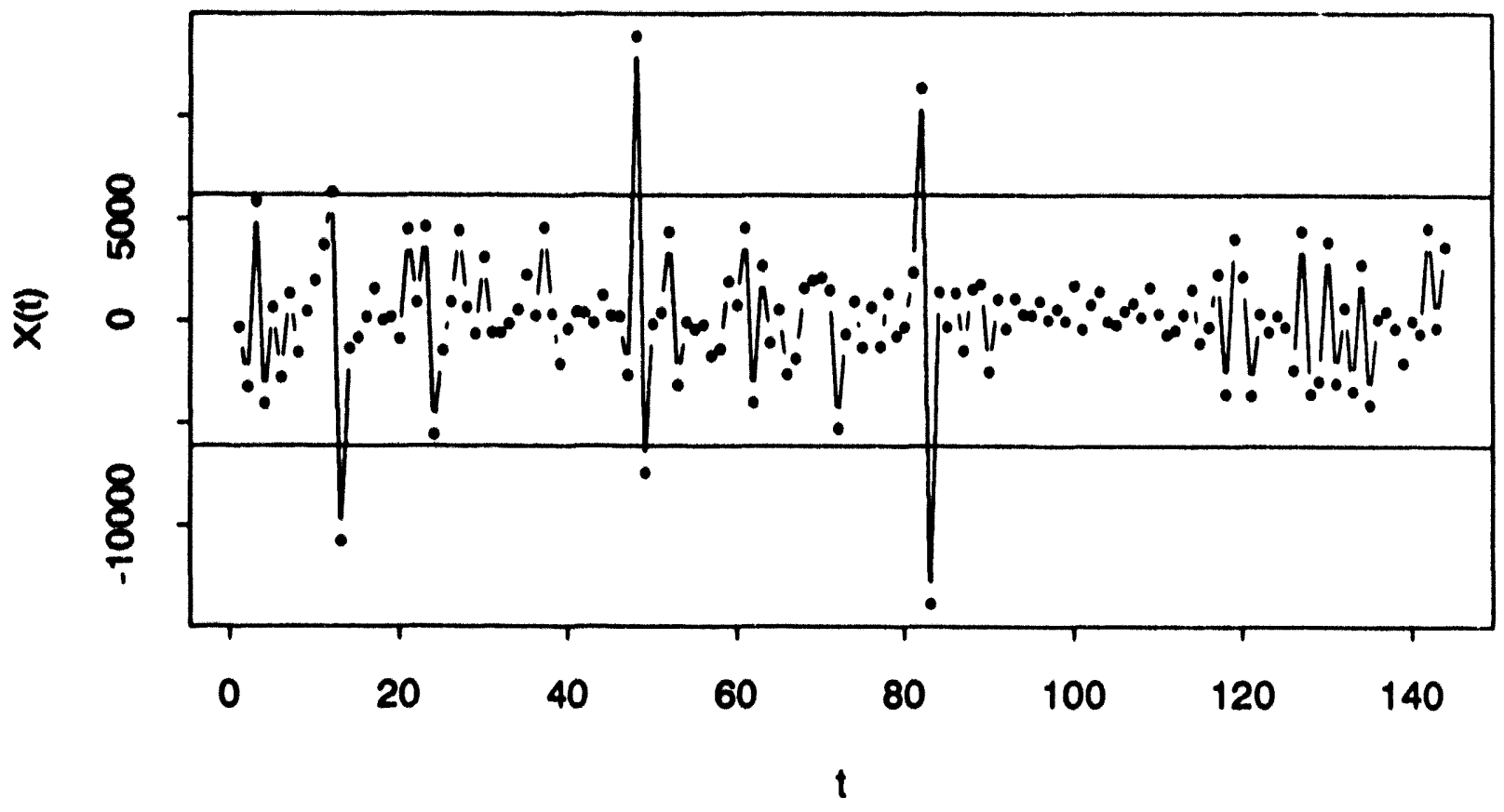

Fig. 18a. Material balance data.

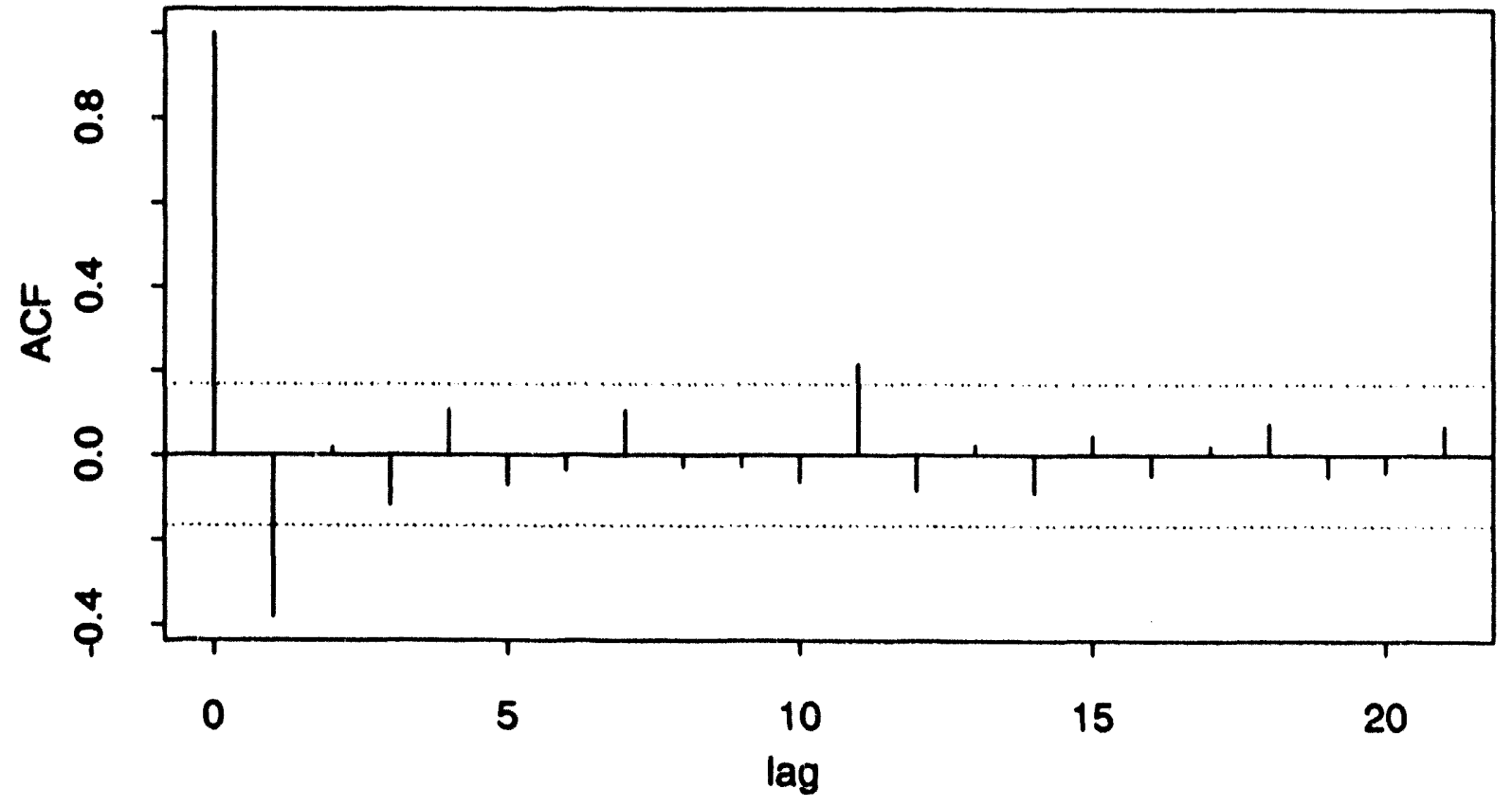

Fig. 18b. ACF versus lag of $X(t)$. 


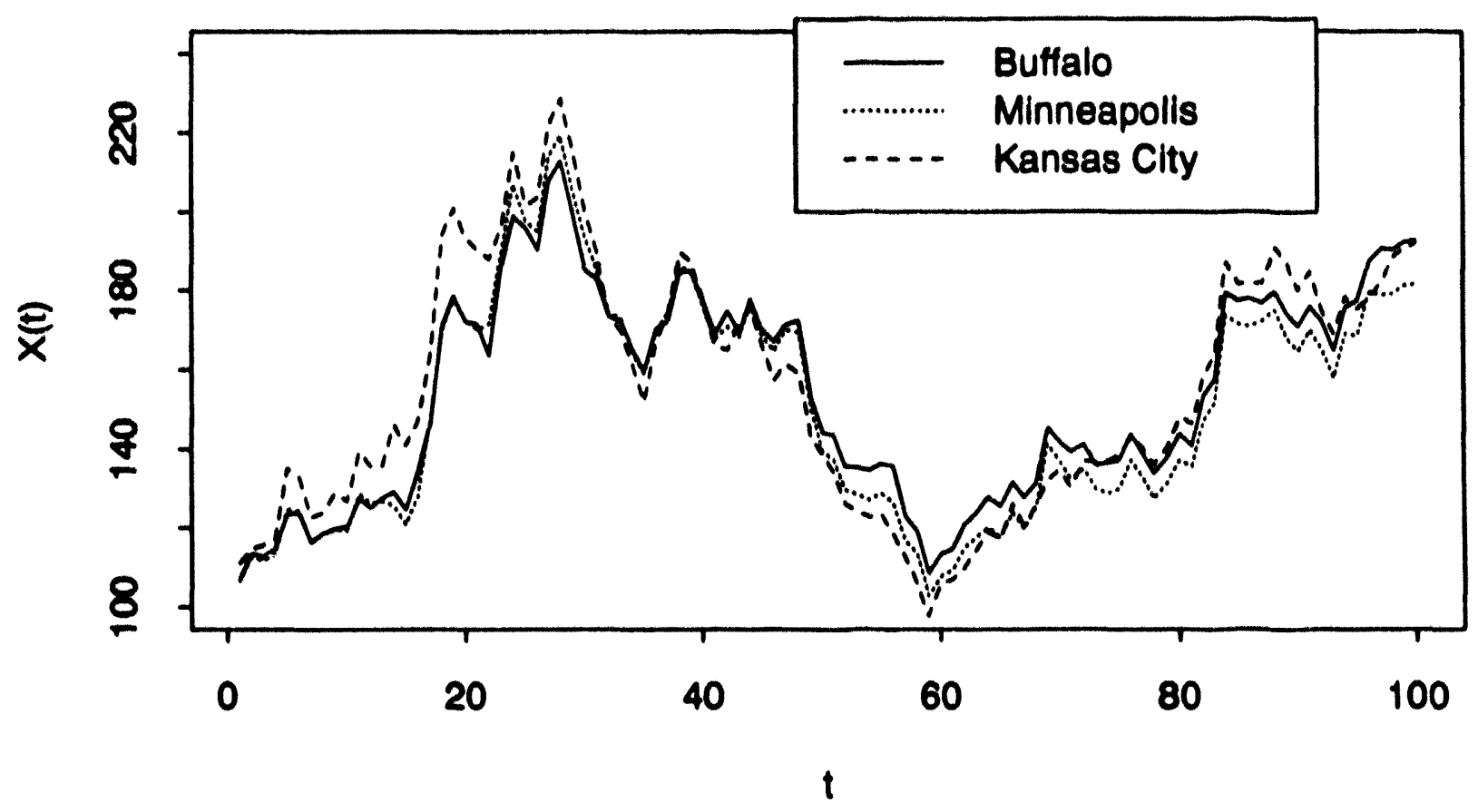

Fig. 19. Flour prices.

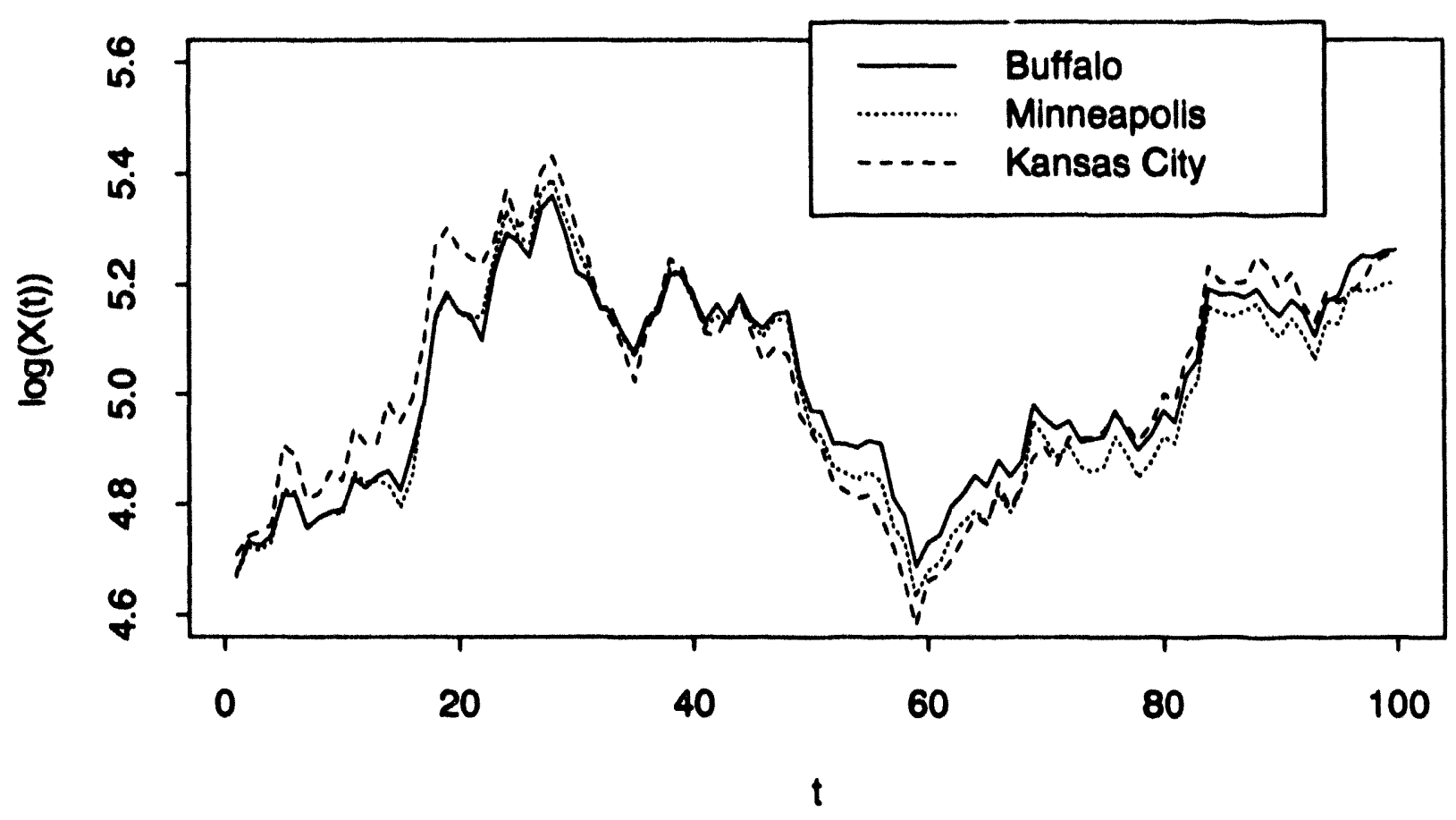

Fig. 19b. Log (flour prices). 


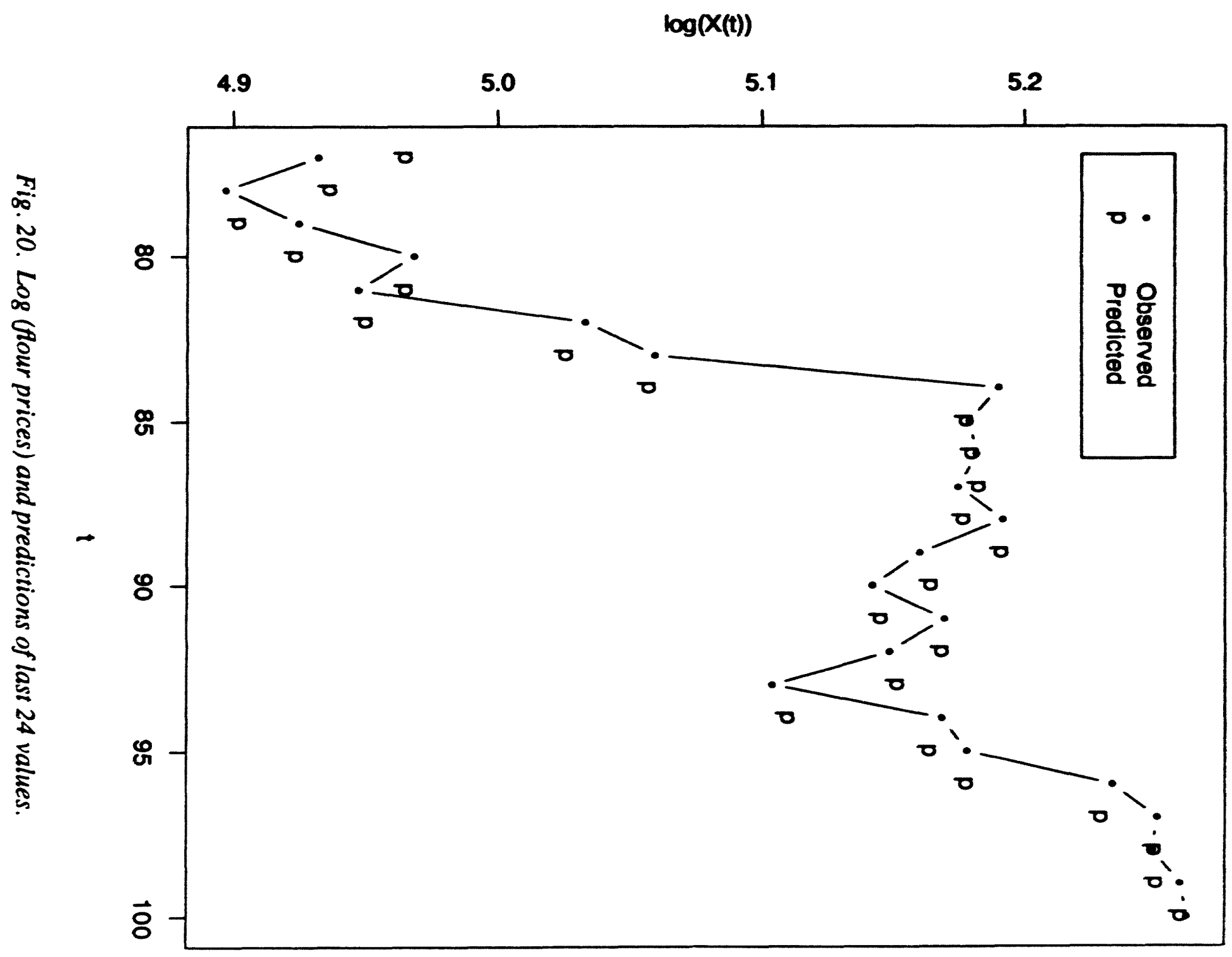



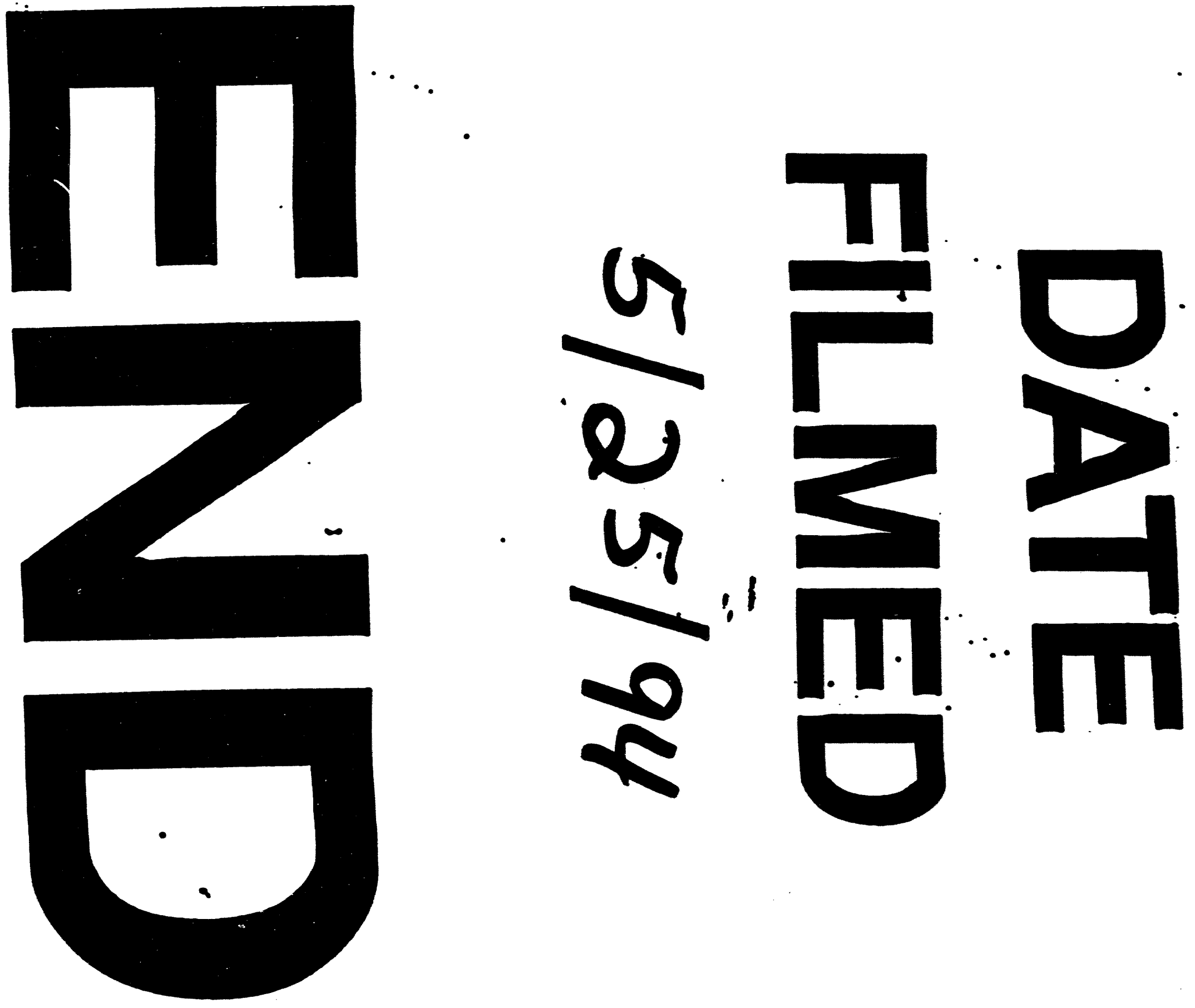
